\title{
Elliptic Problems for the Dolbeault Complex *†
}

\author{
A. Kytmanov \\ S. Myslivets ${ }^{\S}$ \\ B.-W. Schulze \\ N. Tarkhanov
}

May 2, 2001

*AMS subject classification: primary: 32A25; secondary: 35N15, $58 \mathrm{G} 05$.

${ }^{\dagger}$ Key words and phrases: The Dolbeault complex, homotopy formulas, the BochnerMartinelli integral.

$\ddagger$ Supported by the Deutsche Forschungsgemeinschaft

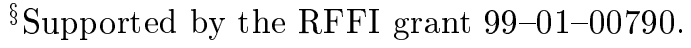




\begin{abstract}
The inhomogeneous $\bar{\partial}$-equation is an inexhaustible source of locally unsolvable equations, subelliptic estimates and other phenomena in partial differential equations. Loosely speaking, for the analysis on complex manifolds with boundary nonelliptic problems are typical rather than elliptic ones. Using explicit integral representations we assign a Fredholm complex to the Dolbeault complex over an arbitrary bounded domain in $\mathbb{C}^{n}$.
\end{abstract}




\section{Contents}

Introduction $\quad 4$

1 Auxiliary results 5

$\begin{array}{llr}2 & \text { A homotopy formula } & 7\end{array}$

$\begin{array}{lll}3 & \text { Algebraic construction } & 10\end{array}$

4 An exact complex 12

5 Fredholm problems $\quad 14$

6 The case $n=1 \quad 16$

$\begin{array}{llr}7 & \text { Identification of } Q^{s}\left(\mathcal{D}, \Lambda^{0,0}\right) & \mathbf{1 7}\end{array}$

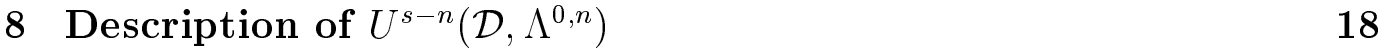

9 Regular domains $\quad 22$

10 Characterisation of $U^{s-q}\left(\mathcal{D}, \Lambda^{0, q}\right) \quad 26$

11 The case $q=n-1 \quad 28$

12 Concluding remarks 33

$\begin{array}{ll}\text { References } & 35\end{array}$ 


\section{Introduction}

Ellipticity of differential (or pseudodifferential) operators is usually formulated as a condition on suitable symbols. It is then expected that the ellipticity entails the Fredholm property in reasonable scales of spaces, e.g., Sobolev spaces.

For operators on a compact closed $C^{\infty}$ manifold this is a well-known relation. There is a natural analogue for complexes, where ellipticity is the exactness of a corresponding complex of symbols, and the Fredholm problem means the finiteness of the associated Euler characteristic.

Standard complexes such as the de Rham and the Dolbeault complexes belong to the latter category, and it is well known, see Atiyah and Bott [AB67], that they possess a parametrix in the class of elliptic pseudodifferential complexes.

The case of a compact $C^{\infty}$ manifold with boundary is completely different. If a certain topological obstruction for the principal symbols vanishes, $\mathrm{cf}$. Atiyah and Bott [AB64], there are elliptic boundary conditions in the sense of the Lopatinskii condition. Associated boundary value problems are again Fredholm in standard Sobolev spaces, cf. also [BdM71].

On the other hand, the Cauchy-Riemann operator in one complex variable and many other interesting geometric operators on a manifold with boundary are elliptic, but they do not admit any elliptic boundary value problems in that sense.

The program to study boundary value problems without Lopatinskii condition has been stimulated by the paper of Atiyah, Patodi and Singer [APS75], who represented the signature of a Riemannian manifold as the index of a Dirac-type operator under nonlocal boundary conditions. The Fredholm theory of such operators was actually well known in another language by the work of Kondrat'ev [Kon67] in the context of operators on a manifold with conical singularities.

It was Calderón [Cal63] who first suggested a more general approach to boundary value problems. Given any elliptic differential operator $A$, the Cauchy data on the boundary for any solution to $A u=0$ uniquely determine $u$ up to a finite-dimensional effect. Hence it follows that any Fredholm operator in the space of Cauchy data leads to a Fredholm boundary value problem for the initial equation $A u=0$, too.

In fact, the Cauchy data spaces give rise to various function spaces in the domain, not necessarily to Sobolev spaces. An example are the generalised Hardy spaces.

Also Seeley's paper [See69] belongs to this development, as well as a calculus of Schulze [Schu01] who completed the set of such problems to a pseudodifferential algebra, where ellipticity does not require the Lopatinskii condition. 
This algebra contains as a very particular case the algebra of Toeplitz operators on the boundary, cf. [Ven72].

The crucial step in the construction consists of specifying the index element of the virtual index bundle on the cosphere bundle of the boundary, induced by the boundary symbol. In general, this may be a hard problem in concrete cases.

The Dolbeault complex belongs to the examples, where the virtual index bundle from the boundary symbol is not a pull-back under the canonical projection of any bundle on the boundary. In other words, in contrast to Fredholm boundary value problems for complexes with Lopatinskii condition, cf. Dynin [Dyn72], Pillat and Schulze [PS80], or Rempel and Schulze [RS82], we may look for alternative constructions of Fredholm complexes associated with the Dolbeault complex.

This is just the program of the present paper. We prove that any homotopy formula for the Dolbeault complex over a bounded domain in $\mathbb{C}^{n}$ with smooth boundary leads to a Fredholm complex. While using a special convolution homotopy formula of Koppelman [Kop67], we actually show that the same ideas work for more general Leray-type integral formulas in strictly pseudoconvex domains in $\mathbb{C}^{n}$.

\section{Auxiliary results}

Let us introduce necessary notation. We will consider the space $\mathbb{C}^{n}$ of complex variables $z=\left(z_{1}, \ldots, z_{n}\right)$. Write $z_{j}=x_{j}+\imath y_{j}$ for $j=1, \ldots, n$. The orientation of $\mathbb{C}^{n}$ is determined by the order of coordinates $\left(x_{1}, \ldots, x_{n}, y_{1}, \ldots, y_{n}\right)$. Then the volume form in $\mathbb{C}^{n}$ is

$$
\begin{aligned}
d v & =d x \wedge d y \\
& =\left(\frac{\imath}{2}\right)^{n} d z \wedge d \bar{z}
\end{aligned}
$$

where $d z=d z_{1} \wedge \ldots \wedge d z_{n}$.

From now on $\mathcal{D}$ is a bounded domain with $\mathbb{C}^{\infty}$ boundary in $\mathbb{C}^{n}$, such that $\mathbb{C}^{n} \backslash \overline{\mathcal{D}}$ is connected. We give $\mathcal{D}$ by $\mathcal{D}=\left\{z \in \mathbb{C}^{n}: \varrho(z)<0\right\}$, where $\varrho$ is a real-valued function of class $C^{\infty}\left(\mathbb{C}^{n}\right)$ with $\nabla \varrho \neq 0$ on $\partial \mathcal{D}$. The function $\varrho$ can be chosen in such a way that $|\nabla \varrho|=1$ on $\partial \mathcal{D}$.

Recall some properties of the Hodge star operator relative to the usual Euclidean metric in $\mathbb{C}^{n}$, cf. [Wel73]. Let $u$ be a differential form of bidegree $(p, q)$ having the form

$$
u=\sum_{I} \sum_{J} u_{I, J}(z) d z_{I} \wedge d \bar{z}_{J}
$$


where the sums are over all increasing multi-indices

$$
\begin{aligned}
& I=\left(i_{1}, \ldots, i_{p}\right), \\
& J=\left(j_{1}, \ldots, j_{q}\right)
\end{aligned}
$$

of integers $1, \ldots, n$, i.e.,

$$
\begin{aligned}
& 1 \leq i_{1}<\ldots<i_{p} \leq n \\
& 1 \leq j_{1}<\ldots<j_{q} \leq n
\end{aligned}
$$

and

$$
\begin{aligned}
d z_{I} & =d z_{i_{1}} \wedge \ldots \wedge d z_{i_{p}} \\
d \bar{z}_{J} & =d \bar{z}_{j_{1}} \wedge \ldots \wedge d \bar{z}_{j_{q}} .
\end{aligned}
$$

Then

$$
\begin{aligned}
\star u & =\sum_{I} \sum_{J} u_{I, J}(z) \star\left(d z_{I} \wedge d \bar{z}_{J}\right) \\
\star\left(d z_{I} \wedge d \bar{z}_{J}\right) & =(-1)^{p n} 2^{p+q}\left(\frac{\imath}{2}\right)^{n} \sigma(I) \sigma(J) d z[J] \wedge d \bar{z}[I]
\end{aligned}
$$

where $d z[J]$ is obtained from $d z$ by removing the differentials $d z_{j_{1}}, \ldots, d z_{j_{q}}$, and $\sigma(J)= \pm 1$ is defined by $d z_{J} \wedge d z[J]=\sigma(J) d z$.

The form $\star u$ is thus a differential form of bidegree $(n-q, n-p)$. Moreover, we have

1) $\star \star u=(-1)^{p+q} u$;

2) $d z_{I} \wedge d \bar{z}_{J} \wedge \star \overline{\left(d z_{I} \wedge d \bar{z}_{J}\right)}=2^{p+q} d v$.

If $u$ and $v$ are differential forms of bidegree $(p, q)$ with coefficients of the Lebesgue class $L^{2}(\mathcal{D})$ then their Hodge scalar product is defined by

$$
(u, v)=\int_{\mathcal{D}} u \wedge \star \bar{v}
$$

so that $\|u\|^{2}=(u, u)$.

Using the Hodge star operator one can explicitly write down the operators $\bar{\partial}^{*}$ and $\partial^{*}$ formally adjoint to $\bar{\partial}$ and $\partial$, respectively:

$$
\begin{aligned}
& \bar{\partial}^{*}=-\star \partial \star \\
& \partial^{*}=-\star \bar{\partial} \star
\end{aligned}
$$

For $s \in \mathbb{Z}_{+}$, we denote by $H^{s}(\mathcal{D})$ the Sobolev space of functions whose derivatives up to order $s$ belong to $L^{2}(\mathcal{D})$. More generally, we write $H^{s}\left(\mathcal{D}, \Lambda^{p, q}\right)$ for the space of all differential forms $u$ of bidegree $(p, q)$ in $\mathcal{D}$, whose coefficients lie in $H^{s}(\mathcal{D})$. These are Hilbert spaces under standard Hermitian structures. Since the operator $\bar{\partial}$ does not change the degree of $u$ in $z$, we restrict our 
discussion to differential forms of bidegree $(0, q)$. For $q=0$ we clearly have $H^{s}\left(\mathcal{D}, \Lambda^{0,0}\right)=H^{s}(\mathcal{D})$.

Given any differential form $u$ of bidegree $(p, q)$ with continuous coefficients in $\overline{\mathcal{D}}$, one says that the tangential part of $u$ on $\partial \mathcal{D}$ is equal to zero (write $\tau(u)=0)$ if

$$
\int_{\partial \mathcal{D}} u \wedge g=0
$$

for all $(n-p, n-q-1)$-forms $g$ with $C^{\infty}$ coefficients in $\overline{\mathcal{D}}$. This just amounts to saying that $u \wedge \bar{\partial} \varrho=0$ on $\partial \mathcal{D}$.

Furthermore, the normal part of $u$ on $\partial \mathcal{D}$ is said to vanish (write $\nu(u)=0$ ) if $\tau(\star u)=0$.

\section{Lemma 1.1}

1) $\tau(u)=0$ if and only if $(\bar{\partial} u, g)=\left(u, \bar{\partial}^{*} g\right)$ for all $g \in C^{\infty}\left(\overline{\mathcal{D}}, \Lambda^{0, n-q-1}\right)$.

2) $\nu(u)=0$ if and only if $\left(\bar{\partial}^{*} u, g\right)=(u, \bar{\partial} g)$ for all $g \in C^{\infty}\left(\overline{\mathcal{D}}, \Lambda^{0, n-q+1}\right)$.

Proof. This follows from Stokes' formula.

\section{A homotopy formula}

The formula of Koppelman [Kop67] for differential forms will be of crucial role in our considerations.

Denote by $g(\zeta, z)$ the standard fundamental solution of the Laplace equation in $\mathbb{C}^{n}$,

$$
g(\zeta, z)= \begin{cases}-\frac{(n-2) !}{(2 \pi \imath)^{n}} \frac{1}{|\zeta-z|^{2 n-2}} & \text { if } n>1, \\ \frac{1}{2 \pi \imath} \log |\zeta-z|^{2} & \text { if } n=1,\end{cases}
$$

and set

$$
U_{0, q+1}(\zeta, z)=\sum_{J}\left(\sum_{j \notin J} \sigma(j, J) \frac{\partial g}{\partial \zeta_{j}} d \bar{\zeta}[j, J] \wedge d \zeta\right) d \bar{z}_{J}
$$

where the sum is over all increasing multi-indices $J=\left(j_{1}, \ldots, j_{q}\right)$ of integers $1, \ldots, n$, and the constant $\sigma(j, J)= \pm 1$ is uniquely determined from the equality

$$
d \bar{\zeta}_{j} \wedge d \bar{\zeta}_{J} \wedge d \bar{\zeta}[j, J]=\sigma(j, J) d \bar{\zeta} .
$$

The kernel $U_{0, q+1}(\zeta, z)$ is iterpreted as a double differential form of bidegree $(n, n-q-1)$ in $\zeta$ and bidegree $(0, q)$ in $z$. For $q=-1$ and $q=n$ we put $U_{0, q+1}(\zeta, z)=0$. 
By $\S 1$ of Aizenberg and Dautov [AD83] the kernel $U_{0, q+1}(\zeta, z)$ can be written in the form

$$
\begin{aligned}
U_{0, q+1}(\zeta, z) & =\partial_{\zeta} g(\zeta, z) \wedge F_{1, q+1}(\zeta, z) \\
F_{1, q+1}(\zeta, z) & =(-1)^{n-q-1} \sum_{J}\left(\sum_{j \notin J} \sigma(j, J)(-1)^{j-1} d \bar{\zeta}[j, J] \wedge d \zeta[j]\right) d \bar{z}_{J}
\end{aligned}
$$

We need yet another form of the kernel $U_{0, q+1}(\zeta, z)$. To this end we introduce the double differential form

$$
V_{0, q+1}(\zeta, z)=\frac{1}{2} \frac{1}{q+1} \sum_{J} \sum_{j \notin J}(\sigma(j \cup J) g(\zeta, z) d \bar{\zeta}[j, J] \wedge d \zeta) d \bar{z}_{j \cup J}
$$

$j \cup J$ being the increasing multi-index of length $q+1$ that is obtained by placing $j$ within $J$.

Lemma 2.1 For $\zeta \neq z$, it follows that

$$
\bar{\partial}_{z}^{*} V_{0, q+1}(\zeta, z)=U_{0, q+1}(\zeta, z) .
$$

Proof. Given any increasing multi-index $K$ of length $q+1$, we get

$$
\begin{aligned}
\bar{\partial}_{z}^{*}\left(g(\zeta, z) d \bar{z}_{K}\right) & =-\star \partial_{z} \star\left(g(\zeta, z) d \bar{z}_{K}\right) \\
& =-2^{q+1}\left(\frac{\imath}{2}\right)^{n} \sigma(K) \star \partial_{z} g(\zeta, z) \wedge d z[K] \wedge d \bar{z} \\
& =2^{q+1}\left(\frac{\imath}{2}\right)^{n} \sigma(K) \star \sum_{k \in K} \frac{\partial g}{\partial \zeta_{k}} d z_{k} \wedge d z[K] \wedge d \bar{z}
\end{aligned}
$$

A trivial verification shows that

$$
\begin{aligned}
d z_{k} \wedge d z[K] & =(-1)^{q} \sigma(k, K \backslash k) \sigma(K \backslash k) d z[K \backslash k] \\
(-1)^{q(n-q)} \sigma(K \backslash k) & =\sigma(\{1, \ldots, n\} \backslash(K \backslash k))
\end{aligned}
$$

whence

$$
\bar{\partial}_{z}^{*}\left(g(\zeta, z) d \bar{z}_{K}\right)=2 \sigma(K) \sum_{k \in K} \sigma(k, K \backslash k) \frac{\partial g}{\partial \zeta_{k}} d \bar{z}_{K \backslash k}
$$

For any multi-index $J$ of length $q$ and $j \notin J$, we can represent the multiindex $K=j \cup J$ in the form $K=k \cup(K \backslash k)$ with $k \in K$ by $q+1$ different 
ways. Hence

$$
\begin{aligned}
\bar{\partial}_{z}^{*} & V_{0, q+1}(\zeta, z) \\
& =\frac{1}{2} \frac{1}{q+1} \sum_{J} \sum_{j \notin J}(\sigma(j \cup J) d \bar{\zeta}[j, J] \wedge d \zeta) \bar{\partial}_{z}^{*}\left(g(\zeta, z) d \bar{z}_{j \cup J}\right) \\
& =\frac{1}{q+1} \sum_{J} \sum_{j \notin J} \sum_{k \in(j \cup J)}\left(\sigma(k,(j \cup J) \backslash k) \frac{\partial g}{\partial \zeta_{k}} d \bar{\zeta}[j, J] \wedge d \zeta\right) d \bar{z}_{(j \cup J) \backslash k} \\
& =U_{0, q+1}(\zeta, z),
\end{aligned}
$$

as desired.

The $\bar{\partial}^{*}$-exactness of the kernel $U_{0, q+1}$ is mentioned in [Tar90, p. 88], however no explicit form of $V_{0, q+1}$ is given.

Lemma 2.2 Away from the diagonal of $\mathbb{C}^{n} \times \mathbb{C}^{n}$, we have

$$
\bar{\partial}_{\zeta} U_{0, q+1}(\zeta, z)=(-1)^{q} \bar{\partial}_{z} U_{0, q}(\zeta, z) .
$$

Proof. Cf. $\S 1$ in [AD83].

In particular, the kernels $U_{0,1}(\zeta, z)$ and $U_{0, n}(\zeta, z)$ are $\bar{\partial}$-closed in $\zeta$ and $z$, respectively, for $\zeta \neq z$.

Consider the bounded operators

$$
\begin{aligned}
M & : \quad H^{s}\left(\mathcal{D}, \Lambda^{0, q}\right) \rightarrow H^{s}\left(\mathcal{D}, \Lambda^{0, q}\right), \\
T & : \quad H^{s}\left(\mathcal{D}, \Lambda^{0, q}\right) \rightarrow H^{s+1}\left(\mathcal{D}, \Lambda^{0, q-1}\right)
\end{aligned}
$$

defined by

$$
\begin{aligned}
(M u)(z) & =\int_{\partial \mathcal{D}} u(\zeta) \wedge U_{0, q+1}(\zeta, z), \\
(T u)(z) & =-\int_{\mathcal{D}} u(\zeta) \wedge U_{0, q}(\zeta, z)
\end{aligned}
$$

for $z \in \mathcal{D}$. The operators $M$ and $T$ are bounded, for the former is a derivative of the simple layer potential and the latter is a derivative of the volume potential in $\mathbb{C}^{n}$.

The differential forms $(M u)(z)$ and $(T u)(z)$ are defined for $z \in \mathbb{C}^{n} \backslash \overline{\mathcal{D}}$, too. We write $(M u)^{ \pm}$and $(T u)^{ \pm}$for the restrictions of $M u$ and $T u$ to $\mathcal{D}$ and $\mathbb{C}^{n} \backslash \overline{\mathcal{D}}$, respectively.

Note that the differential form $M u$ has harmonic coefficients away from the boundary of $\mathcal{D}$, and it decreases as $|z|^{1-2 n}$ at the point at infinity. The 
differential form $T u$ has harmonic coefficients away from the closure of $\mathcal{D}$, and it decreases as $|z|^{1-2 n}$ at the point at infinity.

Having disposed of these preliminary steps we are in a position to formulate the formula of Koppelman [Kop67], cf. also [AD83].

Lemma 2.3 For any $u \in H^{1}\left(\mathcal{D}, \Lambda^{0, q}\right)$, we have

$$
M u+T \bar{\partial} u+\bar{\partial} T u= \begin{cases}u(z) & \text { if } z \in \mathcal{D}, \\ 0 & \text { if } z \in \mathbb{C}^{n} \backslash \overline{\mathcal{D}}\end{cases}
$$

Proof. For those differential forms $u$ which are smooth in the closure of $\mathcal{D}$ this formula is proved in [AD83]. Given any $u \in H^{1}\left(\mathcal{D}, \Lambda^{0, q}\right)$, we choose a sequence $\left\{u_{\nu}\right\}$ of smooth forms approximating $u$ in the Sobolev norm. Writing every $u_{\nu}$ by formula (2.2) and letting $\nu \rightarrow \infty$ we get this formula for $u$ by continuity.

When $q=0,(2.2)$ is nothing else than the Bochner-Martinelli formula for smooth functions.

\section{Algebraic construction}

Let

$$
\begin{aligned}
& 0 \longrightarrow V^{0} \stackrel{A}{\longrightarrow} V^{1} \stackrel{A}{\longrightarrow} \ldots \stackrel{A}{\longrightarrow} V^{N} \longrightarrow 0, \\
& 0 \longrightarrow Q^{0} \stackrel{B}{\longrightarrow} Q^{1} \stackrel{B}{\longrightarrow} \ldots \stackrel{B}{\longrightarrow} Q^{N} \longrightarrow 0
\end{aligned}
$$

be two complexes of Banach spaces. By a cochain mapping of these complexes is meant any sequence of mappings

$$
C^{q}: V^{q} \rightarrow Q^{q}
$$

such that the diagram

$$
\begin{aligned}
& 0 \longrightarrow V^{0} \stackrel{A}{\rightarrow} V^{1} \stackrel{A}{\longrightarrow} \ldots \stackrel{A}{\longrightarrow} V^{N} \longrightarrow 0 \\
& 0 \longrightarrow Q^{0} \stackrel{B}{\longrightarrow} Q^{1} \stackrel{B}{\longrightarrow} \ldots \stackrel{B}{\longrightarrow} Q^{N} \longrightarrow 0
\end{aligned}
$$

commutes, i.e., $C^{q} A=B C^{q-1}$ for all $q$.

Given any cochain mapping $C=\left\{C^{q}\right\}$, one defines a new complex

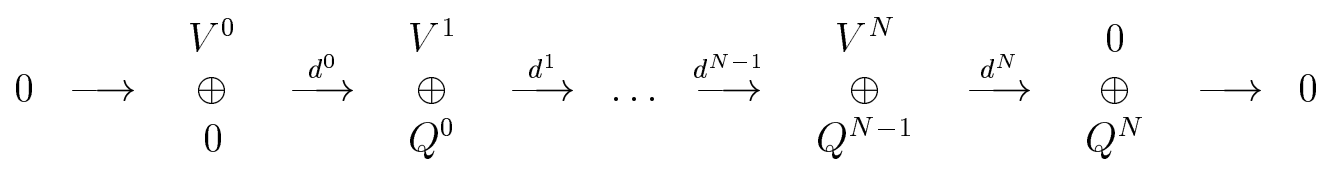


where

$$
d^{q}=\left(\begin{array}{cc}
-A^{q} & 0 \\
C^{q} & B^{q-1}
\end{array}\right) .
$$

It is called the cone of the cochain mapping $C$ and denoted by $\mathcal{C}(C)$, cf. [Spa66] and elsewhere.

By $C^{q} A=B C^{q-1}$ we see that $A^{q}$ restricts to a mapping $\operatorname{ker} C^{q} \rightarrow \operatorname{ker} C^{q+1}$, and $B^{q}$ lifts to a mapping coker $C^{q} \rightarrow \operatorname{coker} C^{q+1}$. We thus get two associated complexes

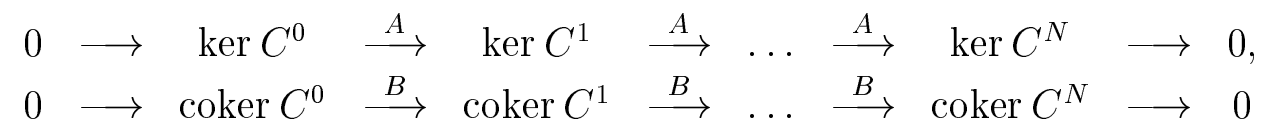

denoted by $\operatorname{ker} C$ and coker $C$, respectively.

Lemma 3.1 Complex (3.2) is Fredholm if and only if so are both the complexes (3.3). In this case

$$
\chi_{\mathcal{C}(C)}=\chi_{\operatorname{ker} C}-\chi_{\operatorname{coker} C} \cdot
$$

Given a (cochain) complex $V$, we write $\chi_{V}$ for the Euler characteristic of $V$, i.e, for the alternating sum of dimensions of the cohomology spaces of $V$, if defined.

Proof. A trivial verification shows that

$$
\begin{aligned}
H^{0}(\mathcal{C}(C)) & =H^{0}(\operatorname{ker} C), \\
H^{q}(\mathcal{C}(C)) & =H^{q}(\operatorname{ker} C) \oplus H^{q-1}(\operatorname{coker} C), \\
H^{N+1}(\mathcal{C}(C)) & =H^{N}(\operatorname{coker} C),
\end{aligned}
$$

and the lemma follows (cf. Proposition 5 in [RS82, 3.2.3.1]).

In particular, complex (3.2) is exact if and only if so are both the complexes (3.3).

We apply the construction of $\mathcal{C}(C)$ in the case when

$$
Q^{q}=V^{q} / U^{q},
$$

$U^{q}$ being closed subspaces of $V^{q}$, such that $A$ maps $U^{q}$ to $U^{q}$ for each $q$. Then $A$ lifts in a natural way to a mapping $Q^{q} \rightarrow Q^{q+1}$ which we denote by $A_{Q}$. By the very definition it fulfills $C^{q} A=A_{Q} C^{q-1}$ where $C^{q}$ are the canonical mappings. Hence we arrive at the complex

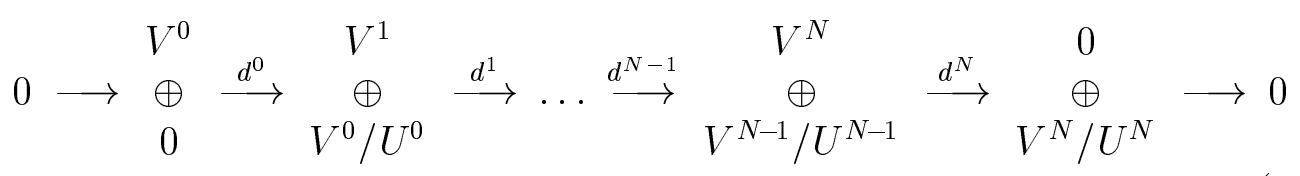


with

$$
d^{q}=\left(\begin{array}{rc}
-A^{q} & 0 \\
C^{q} & A_{Q}^{q-1}
\end{array}\right)
$$

Theorem 3.2 The complex (3.4) is Fredholm if and only if so is the complex

$$
0 \longrightarrow U^{0} \stackrel{A}{\longrightarrow} U^{1} \stackrel{A}{\longrightarrow} \ldots \stackrel{A}{\longrightarrow} U^{N} \longrightarrow 0 .
$$

In this case the Euler characteristic of (3.4) is equal to that of the complex (3.5).

Proof. It suffices to combine Lemma 3.1 with the observation that in our case the complex $\operatorname{ker} C$ reduces to (3.5), and the complex coker $C$ is zero.

\section{An exact complex}

Given any $s=1,2, \ldots$, we introduce the spaces

$$
U^{s}\left(\mathcal{D}, \Lambda^{0, q}\right)=\left\{u \in H^{s}\left(\mathcal{D}, \Lambda^{0, q}\right): M T^{j} u=0 \text { in } \mathcal{D} \text { for all } j \in \mathbb{Z}_{+}\right\} .
$$

Since both $M$ and $T$ are continuous mappings $U^{s}\left(\mathcal{D}, \Lambda^{0, q}\right)$ is a closed subspace of $H^{s}\left(\mathcal{D}, \Lambda^{0, q}\right)$, hence a Hilbert space. We give a description of these spaces later.

In the meanwhile we note that the operator $T$ decreases the degree of the form in $z$ by 1 . It follows $T^{j} u \equiv 0$ for $j>q$. Hence the equalities $M T^{j} u=0$ for all $j \in \mathbb{Z}_{+}$can be formally summarised by saying that $M(I-T)^{-1} u \equiv 0$ in $\mathcal{D}, I$ being the identity operator.

Theorem 4.1 Suppose $s \geq n$. The following sequence of Hilbert spaces is an exact complex:

$$
0 \longrightarrow U^{s}\left(\mathcal{D}, \Lambda^{0,0}\right) \stackrel{\bar{\partial}}{\longrightarrow} U^{s-1}\left(\mathcal{D}, \Lambda^{0,1}\right) \stackrel{\bar{\partial}}{\longrightarrow} \ldots \stackrel{\bar{\partial}}{\longrightarrow} U^{s-n}\left(\mathcal{D}, \Lambda^{0, n}\right) \longrightarrow 0 .
$$

Proof. We begin by verifying that the operator $\bar{\partial}$ preserves the above spaces, i.e., it restricts to a mapping $U^{s-q}\left(\mathcal{D}, \Lambda^{0, q}\right) \rightarrow U^{s-q-1}\left(\mathcal{D}, \Lambda^{0, q+1}\right)$ for all $q$.

Let $u \in U^{s-q}\left(\mathcal{D}, \Lambda^{0, q}\right)$. Then $M T^{j} u=0$ in $\mathcal{D}$ for all $j=0,1, \ldots, q$. Set $f=\bar{\partial} u$.

The operators $\bar{\partial}$ and $M$ commute. Indeed, for $z \notin \partial \mathcal{D}$ we get

$$
\bar{\partial}(M u)(z)=\int_{\partial \mathcal{D}} u(\zeta) \wedge \bar{\partial}_{z} U_{0, q+1}(\zeta, z)
$$




$$
\begin{aligned}
& =(-1)^{q+1} \int_{\partial \mathcal{D}} u(\zeta) \wedge \bar{\partial}_{\zeta} U_{0, q+2}(\zeta, z) \\
& =\int_{\partial \mathcal{D}} \bar{\partial} u(\zeta) \wedge U_{0, q+2}(\zeta, z) \\
& =(M \bar{\partial} u)(z),
\end{aligned}
$$

the second equality being due to Lemma 2.2. Hence it follows that

$$
\begin{aligned}
M f & =\bar{\partial} M u \\
& =0
\end{aligned}
$$

in $\mathcal{D}$.

Consider $M T f$. By formula (2.2) we get $u=T \bar{\partial} u+\bar{\partial} T u$ in $\mathcal{D}$. It follows that

$$
\begin{aligned}
0 & =M u \\
& =M T \bar{\partial} u+M \bar{\partial} T u \\
& =M T f+\bar{\partial} M T u
\end{aligned}
$$

whence $M T f=0$ in $\mathcal{D}$.

We next show that

$$
M T^{j} \bar{\partial} u=-M T^{j-1} \bar{\partial} T u
$$

for each $j=1, \ldots, q+1$. To this end, we apply the operator $T^{j-1}$ to both sides of formula (2.2). This gives

$$
T^{j-1} u=T^{j} \bar{\partial} u+T^{j-1} \bar{\partial} T u
$$

whence

$$
M T^{j-1} u=M T^{j} \bar{\partial} u+M T^{j-1} \bar{\partial} T u .
$$

Since $M T^{j-1} u \equiv 0$ in $\mathcal{D}$, we get (4.2), as desired.

We now proceed by induction

$$
\begin{aligned}
M T^{j} f & =M T^{j} \bar{\partial} u \\
& =-M T^{j-1} \bar{\partial} T u \\
& =\cdots \\
& =(-1)^{j} M \bar{\partial} T^{j} u \\
& =(-1)^{j} \bar{\partial} M T^{j} u
\end{aligned}
$$

for $j=2, \ldots, q+1$. Since $M T^{j} u \equiv 0$ in $\mathcal{D}$, we deduce that $M T^{j} f \equiv 0$ for all $j \in \mathbb{Z}_{+}$. 
We have thus proved that $\bar{\partial} u \in U^{s-q-1}\left(\mathcal{D}, \Lambda^{0, q+1}\right)$. In other words, the complex (4.1) is well defined.

Let us prove that (4.1) is exact. Suppose $u \in U^{s}\left(\mathcal{D}, \Lambda^{0,0}\right)$ and $\bar{\partial} u=0$ in $\mathcal{D}$. Then $u$ is a holomorphic function in $\mathcal{D}$. Since $M u=0$ in $\mathcal{D}$, the BochnerMartinelli formula yields $u=M u=0$ in $\mathcal{D}$. Hence $(4.1)$ is exact at step $q=0$.

Let $0<q<n$ and let $f \in U^{s-q}\left(\mathcal{D}, \Lambda^{0, q}\right)$ satisfy $\bar{\partial} f=0$ in $\mathcal{D}$. By formula (2.2) we get

$$
\begin{aligned}
f & =M f+T \bar{\partial} f+\bar{\partial} T f \\
& =\bar{\partial} u
\end{aligned}
$$

where $u=T f$. The boundedness of $T$ implies $u \in H^{s-q+1}\left(\mathcal{D}, \Lambda^{0, q-1}\right)$. On the other hand, from $M T^{j} f=0$ in $\mathcal{D}$ for all $j \in \mathbb{Z}_{+}$it follows that the same is true for $u=T f$. Hence $u \in U^{s-q+1}\left(\mathcal{D}, \Lambda^{0, q-1}\right)$, which shows the exactness of (4.1) at step $q$.

Finally, pick $f \in U^{s-n}\left(\mathcal{D}, \Lambda^{0, n}\right)$. The conditions $M f \equiv 0$ and $\bar{\partial} f \equiv 0$ in $\mathcal{D}$ are automatically fulfilled in this case. By formula (2.2) we obtain $f=\bar{\partial} u$ with $u=T f$. Analysis similar to that at step $q$ shows that $u \in U^{s-n+1}\left(\mathcal{D}, \Lambda^{0, n-1}\right)$, and the proof is complete.

\section{$5 \quad$ Fredholm problems}

For $q=0,1, \ldots, n$ and $s \geq n$, we consider the quotient spaces

$$
Q^{s-q}\left(\mathcal{D}, \Lambda^{0, q}\right)=\frac{H^{s-q}\left(\mathcal{D}, \Lambda^{0, q}\right)}{U^{s-q}\left(\mathcal{D}, \Lambda^{0, q}\right)}
$$

under the quotient topology. Using the Hilbert structure in $H^{s-q}\left(\mathcal{D}, \Lambda^{0, q}\right)$ we might specify $Q^{s-q}\left(\mathcal{D}, \Lambda^{0, q}\right)$ as the orthogonal complement of $U^{s-q}\left(\mathcal{D}, \Lambda^{0, q}\right)$ in $H^{s-q}\left(\mathcal{D}, \Lambda^{0, q}\right)$.

Denote by $C^{q}$ the canonical mapping $H^{s-q}\left(\mathcal{D}, \Lambda^{0, q}\right)$ to $Q^{s-q}\left(\mathcal{D}, \Lambda^{0, q}\right)$. Since $\bar{\partial}$ fulfills

$$
\begin{array}{ll}
\bar{\partial}: & H^{s-q}\left(\mathcal{D}, \Lambda^{0, q}\right) \rightarrow H^{s-q-1}\left(\mathcal{D}, \Lambda^{0, q+1}\right), \\
\bar{\partial}: & U^{s-q}\left(\mathcal{D}, \Lambda^{0, q}\right) \rightarrow U^{s-q-1}\left(\mathcal{D}, \Lambda^{0, q+1}\right),
\end{array}
$$

the second property being a consequence of Theorem 4.1, it lifts to a continuous mapping

$$
\bar{\partial}_{Q}: Q^{s-q}\left(\mathcal{D}, \Lambda^{0, q}\right) \rightarrow Q^{s-q-1}\left(\mathcal{D}, \Lambda^{0, q+1}\right)
$$

for every $q$. Obviously,

$$
\begin{aligned}
\bar{\partial}_{Q} \circ \bar{\partial}_{Q} & =0, \\
C \bar{\partial} & =\bar{\partial}_{Q} C .
\end{aligned}
$$


The algebraic construction of the operator $\bar{\partial}_{Q}$ is actually the same as that of the tangential Cauchy-Riemann operator $\bar{\partial}_{b}$, cf. [KR65].

Applying the algebraic construction (3.4) we thus obtain a complex of Banach spaces

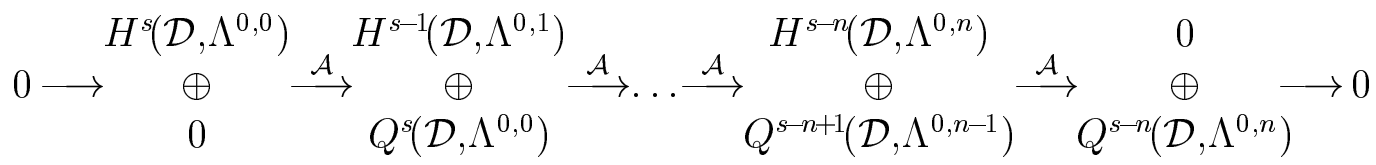

with

$$
\mathcal{A}=\left(\begin{array}{rr}
-\bar{\partial} & 0 \\
C & \bar{\partial}_{Q}
\end{array}\right)
$$

Theorem 5.1 Suppose $n>1$. Then for each $s \geq n$ the sequence (5.2) is an exact complex.

Proof. The sequence (5.2) is a complex, for

$$
\begin{aligned}
\mathcal{A} \circ \mathcal{A} & =\left(\begin{array}{rr}
-\bar{\partial} & 0 \\
C & \bar{\partial}_{Q}
\end{array}\right)\left(\begin{array}{cc}
-\bar{\partial} & 0 \\
C & \bar{\partial}_{Q}
\end{array}\right) \\
& =\left(\begin{array}{cc}
\bar{\partial} \circ \bar{\partial} & 0 \\
-C \bar{\partial}+\bar{\partial}_{Q} C & \bar{\partial}_{Q} \circ \bar{\partial}_{Q}
\end{array}\right) \\
& =0
\end{aligned}
$$

by $(5.1)$.

Let $u_{1} \in H^{s}\left(\mathcal{D}, \Lambda^{0,0}\right)$ and

$$
\mathcal{A}\left(\begin{array}{c}
u_{1} \\
0
\end{array}\right)=0
$$

then $-\bar{\partial} u_{1}=0$ and $u_{1} \in U^{s}\left(\mathcal{D}, \Lambda^{0,0}\right)$. This just amounts to saying that $u_{1}$ is holomorphic in $\mathcal{D}$ and $M u_{1}=0$ in $\mathcal{D}$. By the Bochner-Martinelli formula, $u_{1} \equiv 0$ in $\mathcal{D}$, showing the exactness of (5.2) at step $q=0$.

Consider the intermediate fragments of the complex (5.2). Suppose

$$
\left(\begin{array}{c}
f_{1} \\
f_{2}
\end{array}\right) \in \begin{gathered}
H^{s-q}\left(\mathcal{D}, \Lambda^{0, q}\right) \\
Q^{s-q+1}\left(\mathcal{D}, \Lambda^{0, q-1}\right)
\end{gathered}
$$

satisfies

$$
\left\{\begin{aligned}
-\bar{\partial} f_{1} & =0 \\
C f_{1}+\bar{\partial}_{Q} f_{2} & =0
\end{aligned}\right.
$$


in $\mathcal{D}$. To shorten notation, we use the same letters for equivalence classes and their representatives. Then an equivalent formulation of the compatibility condition (5.3) is

$$
\left\{\begin{aligned}
-\bar{\partial} f_{1} & =0 \\
f_{1}+\bar{\partial} f_{2} & \in U^{s-q}\left(\mathcal{D}, \Lambda^{0, q}\right) .
\end{aligned}\right.
$$

Since $f_{1}+\bar{\partial} f_{2}$ is $\bar{\partial}$-closed, by Theorem 4.1 there exists a differential form $u_{1} \in U^{s-q+1}\left(\mathcal{D}, \Lambda^{0, q-1}\right)$ such that $\bar{\partial} u_{1}=f_{1}+\bar{\partial} f_{2}$ in $\mathcal{D}$. Setting $u_{2}=0$ we thus obtain

$$
\left\{\begin{aligned}
-\bar{\partial}\left(f_{2}-u_{1}\right) & =f_{1}, \\
C\left(f_{2}-u_{1}\right)+\bar{\partial}_{Q} u_{2} & =f_{2},
\end{aligned}\right.
$$

showing the exactness of (5.2) at step $q$.

Finally we prove the exactness of (5.2) at step $q=n+1$. To this end we pick any $f_{2} \in Q^{s-n}\left(\mathcal{D}, \Lambda^{0, n}\right)$, the compatibility conditions are missing. Passing to the representatives of equivalence classes, we rewrite the inhomogeneous equation

$$
\mathcal{A}\left(\begin{array}{l}
u_{1} \\
u_{2}
\end{array}\right)=\left(\begin{array}{c}
0 \\
f_{2}
\end{array}\right)
$$

in the form

$$
\left\{\begin{aligned}
-\bar{\partial} u_{1} & =0 \\
u_{1}+\bar{\partial} u_{2}-f_{2} & \in U^{s-n}\left(\mathcal{D}, \Lambda^{0, n}\right)
\end{aligned}\right.
$$

in $\mathcal{D}$. By formula $(2.2)$ we get $f_{2}=\bar{\partial} u_{2}$ where $u_{2}=T f_{2}$ belongs to the space $H^{s-n+1}\left(\mathcal{D}, \Lambda^{0, n-1}\right)$. Setting $u_{1}=0$ and $u_{2}=T f_{2}$ we thus derive a solution of the system, as desired.

\section{The case $n=1$}

Consider complex (5.2) in the complex plane, i.e., for $n=1$,

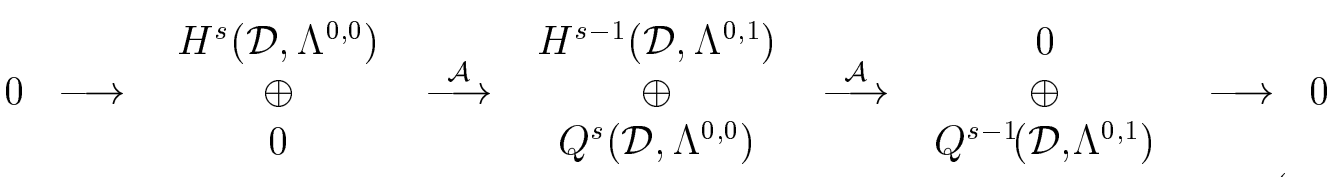

where

$$
\mathcal{A}=\left(\begin{array}{rr}
-\bar{\partial} & 0 \\
C & \bar{\partial}_{Q}
\end{array}\right) .
$$

Let us describe the spaces of this complex. By the Cauchy formula for smooth functions, any $u \in H^{s}\left(\mathcal{D}, \Lambda^{0,0}\right)$ has the form $u=M u+T \bar{\partial} u$ in $\mathcal{D}$. Hence it follows that

$$
Q^{s}\left(\mathcal{D}, \Lambda^{0,0}\right)=\frac{\left\{M u+T \bar{\partial} u: u \in H^{s}\left(\mathcal{D}, \Lambda^{0,0}\right)\right\}}{\left\{T \bar{\partial} u: u \in H^{s}\left(\mathcal{D}, \Lambda^{0,0}\right)\right\}}
$$




$$
=\left\{M u: u \in H^{s}\left(\mathcal{D}, \Lambda^{0,0}\right)\right\},
$$

which is in fact a generalised Hardy space of holomorphic functions in the domain $\mathcal{D}$.

Consider the space

$$
U^{s-1}\left(\mathcal{D}, \Lambda^{0,1}\right)=\left\{u \in H^{s-1}\left(\mathcal{D}, \Lambda^{0,1}\right): M T u=0\right\},
$$

the condition $M u=0$ being automatically fulfilled for all forms $u$ of bidegree $(0,1)$. If $n=1$, both $M$ and $T$ are integral operators with the Cauchy kernel in the complex plane. Therefore, $T u$ is a holomorphic function in the complement of $\overline{\mathcal{D}}$, and it decreases like $1 /|z|$ when $z \rightarrow \infty$. It follows that $M T u=0$ in $\mathcal{D}$ for all $u \in H^{s-1}\left(\mathcal{D}, \Lambda^{0,1}\right)$. We have thus proved that $U^{s-1}\left(\mathcal{D}, \Lambda^{0,1}\right)=H^{s-1}\left(\mathcal{D}, \Lambda^{0,1}\right)$ whence $Q^{s-1}\left(\mathcal{D}, \Lambda^{0,1}\right)=0$.

Summarising, we arrive at an exact complex

$$
0 \longrightarrow H^{s}\left(\mathcal{D}, \Lambda^{0,0}\right) \stackrel{\left(\begin{array}{c}
-\bar{\partial} \\
M
\end{array}\right)}{\longrightarrow} \begin{gathered}
H^{s-1}\left(\mathcal{D}, \Lambda^{0,1}\right) \\
\oplus \\
Q^{s}\left(\mathcal{D}, \Lambda^{0,0}\right)
\end{gathered} \longrightarrow 0
$$

to be obviously interpreted as a Fredholm boundary value problem for the Cauchy-Riemann system on $\mathbb{C}$.

\section{Identification of $Q^{s}\left(\mathcal{D}, \Lambda^{0,0}\right)$}

We now turn to a description of the spaces $U^{s-q}\left(\mathcal{D}, \Lambda^{0, q}\right)$ in order to identify the quotients $Q^{s-q}\left(\mathcal{D}, \Lambda^{0, q}\right)$.

Theorem 7.1 Suppose $n>1$ and $u \in H^{s}\left(\mathcal{D}, \Lambda^{0,0}\right)$. Then $M u=0$ in $\mathcal{D}$ if and only if $u$ vanishes on $\partial \mathcal{D}$. In particular,

$$
Q^{s}\left(\mathcal{D}, \Lambda^{0,0}\right) \cong H^{s-\frac{1}{2}}(\partial \mathcal{D}) .
$$

Proof. If $u$ vanishes on $\partial \mathcal{D}$ then obviously $M u=0$. Conversely, suppose $u \in H^{s}\left(\mathcal{D}, \Lambda^{0,0}\right)$ and $M u=0$ in $\mathcal{D}$. By Corollary 15.6 of [Kyt95] we deduce that $u=M u$ on $\partial \mathcal{D}$, and so $u$ vanishes on the boundary. To prove the second part of the theorem we observe that $H^{s}\left(\mathcal{D}, \Lambda^{0,0}\right)$ is the direct sum of two subspaces. The first of the two is the subspace of $H^{s}\left(\mathcal{D}, \Lambda^{0,0}\right)$ consisting of harmonic functions. The second subspace consists of those functions in $H^{s}\left(\mathcal{D}, \Lambda^{0,0}\right)$ which vanish on the boundary. As the Dirichlet problem in $\mathcal{D}$ is uniquely solvable, the first subspace is topologically isomorphic to $H^{s-1 / 2}(\partial \mathcal{D})$. Hence the theorem follows. 


\section{Description of $U^{s-n}\left(\mathcal{D}, \Lambda^{0, n}\right)$}

In the sequel we need a jump theorem for the boundary integral $M u$ in the formula of Koppelman.

Theorem 8.1 For any differential form $u \in H^{s-q}\left(\mathcal{D}, \Lambda^{0, q}\right), s \geq n$, it follows that

$$
\begin{aligned}
& \tau(M u)^{+}-\tau(M u)^{-}=\tau(u), \\
& \nu(M u)^{+}-\nu(M u)^{-}=0
\end{aligned}
$$

on $\partial \mathcal{D}$.

Proof. Cf. [Kyt95, Tar90].

Pick a differential form $u \in H^{s-n}\left(\mathcal{D}, \Lambda^{0, n}\right)$. It has the form $u=c(z) d \bar{z}$, with $c \in H^{s-n}(\mathcal{D})$. Denote by $G u$ the volume potential of $u$,

$$
\begin{aligned}
G u(z) & =\int_{\mathcal{D}} u(\zeta) \wedge g(\zeta, z) d \zeta \\
& =\int_{\mathcal{D}} c(\zeta) g(\zeta, z) d \bar{\zeta} \wedge d \zeta
\end{aligned}
$$

defined for $z \in \mathbb{C}^{n}$.

Theorem 8.2 Given any form $u \in H^{s-n}\left(\mathcal{D}, \Lambda^{0, n}\right)$, the following are equivalent:

1) $u \in U^{s-n}\left(\mathcal{D}, \Lambda^{0, n}\right)$.

2) $M T u=0$ in $\mathcal{D}$.

3) $T^{2} u=0$ in $\mathbb{C}^{n}$.

4) $\left.G u\right|_{\partial \mathcal{D}}$ extends to an antiholomorphic function in $\mathcal{D}$.

5) $c=\Delta v$ in $\mathcal{D}$, where $v \in H^{s-n+2}(\mathcal{D}) \cap H^{2}\left(\mathbb{C}^{n}\right)$ is harmonic in $\mathbb{C}^{n} \backslash \overline{\mathcal{D}}$ and $\left.v\right|_{\partial \mathcal{D}}$ extends to an antiholomorphic function in $\mathcal{D}$.

The proof falls naturally into a number of lemmas.

Lemma 8.3 In order that $M T u=0$ in $\mathcal{D}$ it is necessary and sufficient that the restriction of $G u$ to $\partial \mathcal{D}$ might be extended to an antiholomorphic function in $\mathcal{D}$.

Proof. Set

$$
P(T u)(z)=\int_{\partial \mathcal{D}}(T u)(\zeta) \wedge g(\zeta, z) d \zeta
$$

for $z \in \mathbb{C}^{n}$ 
We first show that $M(T u)=0$ in $\mathcal{D}$ if and only if $P(T u)$ is antiholomorphic in $\mathcal{D}$. Indeed,

$$
\begin{aligned}
M(T u)(z) & =\int_{\partial \mathcal{D}}(T u)(\zeta) \wedge U_{0, n}(\zeta, z) \\
& =\int_{\partial \mathcal{D}}(T u)(\zeta) \wedge \sum_{j=1}^{n}(-1)^{j-1} \frac{\partial g}{\partial \zeta_{j}}(\zeta, z) d \zeta d \bar{z}[j] \\
& =\sum_{j=1}^{n}(-1)^{j} \frac{\partial}{\partial z_{j}}\left(\int_{\partial \mathcal{D}}(T u)(\zeta) \wedge g(\zeta, z) d \zeta\right) d \bar{z}[j] \\
& =\sum_{j=1}^{n}(-1)^{j} \frac{\partial}{\partial z_{j}}(P(T u)(z)) d \bar{z}[j]
\end{aligned}
$$

for $z \in \mathcal{D}$. If $M(T u)=0$ in $\mathcal{D}$ then $P(T u)$ is antiholomorphic in $\mathcal{D}$, and conversely, as desired.

Consider now the form $P(T u)$. An easy calculation shows that

$$
\begin{aligned}
(T u)(\zeta) \wedge d \zeta & =-\int_{\mathcal{D}} u(w) \wedge U_{0, n}(w, \zeta) \wedge d \zeta \\
& =-\int_{\mathcal{D}} c(w) \sum_{j=1}^{n}(-1)^{j-1} \frac{\partial g}{\partial w_{j}}(w, \zeta) d \bar{w} \wedge d w d \bar{\zeta}[j] \wedge d \zeta \\
& =\sum_{j=1}^{n}(-1)^{j-1} \frac{\partial}{\partial \zeta_{j}}\left(\int_{\mathcal{D}} c(w) g(w, \zeta) d \bar{w} \wedge d w\right) d \bar{\zeta}[j] \wedge d \zeta \\
& =\sum_{j=1}^{n}(-1)^{j-1} \frac{\partial(G u)}{\partial \zeta_{j}}(\zeta) d \bar{\zeta}[j] \wedge d \zeta
\end{aligned}
$$

for all $\zeta \in \mathbb{C}^{n}$. The function $G u$ is of class $H^{2}\left(\mathbb{C}^{n}\right)$ and harmonic in $\mathbb{C}^{n} \backslash \overline{\mathcal{D}}$, hence the differential form $(T u) \wedge d \zeta$ is closed in $\mathbb{C}^{n} \backslash \overline{\mathcal{D}}$, for

$$
\begin{aligned}
d(T u) \wedge d \zeta & =\Delta(G u) d \bar{\zeta} \wedge d \zeta \\
& =0
\end{aligned}
$$

in $\mathbb{C}^{n} \backslash \overline{\mathcal{D}}$.

If $z \in \mathcal{D}$ then

$$
\begin{aligned}
P(T u)(z) & =\int_{\partial \mathcal{D}} g(\zeta, z)(T u)(\zeta) \wedge d \zeta \\
& =-\int_{\mathbb{C}^{n} \backslash \mathcal{D}} \sum_{j=1}^{n} \frac{\partial g}{\partial \bar{\zeta}_{j}}(\zeta, z) \frac{\partial(G u)}{\partial \zeta_{j}}(\zeta) d \bar{\zeta} \wedge d \zeta
\end{aligned}
$$




$$
\begin{aligned}
& =\int_{\partial \mathcal{D}}(G u)(\zeta) \sum_{j=1}^{n}(-1)^{j-1} \frac{\partial g}{\partial \bar{\zeta}_{j}}(\zeta, z) d \bar{\zeta} \wedge d \zeta[j] \\
& =\int_{\partial \mathcal{D}}(G u)(\zeta) \overline{U_{0,1}(\zeta, z)}
\end{aligned}
$$

by Stokes' formula.

By Corollary 15.6 of [Kyt95] it follows that $P(T u)$ is antiholomorphic in $\mathcal{D}$ if and only if $P(T u)=G u$ on $\partial \mathcal{D}$. Thus, the equality $M T u=0$ in $\mathcal{D}$ just amounts to saying that the restriction of $G u$ to $\partial \mathcal{D}$ extends to an antiholomorphic function in $\mathcal{D}$.

Lemma 8.3 yields the equivalence of assertions 2) and 4) in Theorem 8.2.

Lemma 8.4 The equality $M T u=0$ holds in $\mathcal{D}$ if and only if $T^{2} u=0$ in all of $\mathbb{C}^{n}$.

Proof. We first rewrite the kernel $U_{0, n-1}(\zeta, z)$ in a slightly different way. Namely, we put all increasing multi-indices $J$ of length $n-2$ in order

$$
J=(1, \ldots, j-1, j+1, \ldots, k-1, k+1, \ldots, n)
$$

where $1 \leq j<k \leq n$. Then

$$
U_{0, n-1}(\zeta, z)=\sum_{j<k}\left((-1)^{n+j+k-1} \frac{\partial g}{\partial \zeta_{j}} d \bar{\zeta}_{k}+(-1)^{n+j+k} \frac{\partial g}{\partial \zeta_{k}} d \bar{\zeta}_{j}\right) \wedge d \zeta d \bar{z}[j, k]
$$

as is easy to check.

Hence it follows that

$$
\begin{aligned}
& T u(\zeta) \wedge U_{0, n-1}(\zeta, z) \\
& =\sum_{j<k}(-1)^{j+k}\left(\frac{\partial g}{\partial \zeta_{j}} \frac{\partial(G u)}{\partial \zeta_{k}}-\frac{\partial g}{\partial \zeta_{k}} \frac{\partial(G u)}{\partial \zeta_{j}}\right) d \bar{\zeta} \wedge d \zeta d \bar{z}[j, k] \\
& =-\sum_{j<k}(d g(\zeta, z) \wedge d(G u)(\zeta) \wedge d \bar{\zeta} \wedge d \zeta[j, k]) d \bar{z}[j, k]
\end{aligned}
$$

for

$$
\begin{aligned}
& d g(\zeta, z) \wedge d(G u)(\zeta) \wedge d \bar{\zeta} \wedge d \zeta[j, k] \\
& =\left(\frac{\partial g}{\partial \zeta_{j}} d \zeta_{j}+\frac{\partial g}{\partial \zeta_{k}} d \zeta_{k}\right) \wedge\left(\frac{\partial(G u)}{\partial \zeta_{j}} d \zeta_{j}+\frac{\partial(G u)}{\partial \zeta_{k}} d \zeta_{k}\right) \wedge d \bar{\zeta} \wedge d \zeta[j, k] \\
& =(-1)^{j+k-1}\left(\frac{\partial g}{\partial \zeta_{j}} \frac{\partial(G u)}{\partial \zeta_{k}}-\frac{\partial g}{\partial \zeta_{k}} \frac{\partial(G u)}{\partial \zeta_{j}}\right) d \bar{\zeta} \wedge d \zeta
\end{aligned}
$$


whenever $1 \leq j<k \leq n$.

We thus get

$$
T^{2} u(z)=\sum_{j<k}\left(\int_{\mathcal{D}} d g(\zeta, z) \wedge d(G u)(\zeta) \wedge d \bar{\zeta} \wedge d \zeta[j, k]\right) d \bar{z}[j, k]
$$

for all $z \in \mathbb{C}^{n}$. For $z \in \mathbb{C}^{n} \backslash \overline{\mathcal{D}}$ we can moreover invoke Stokes' formula, thus obtaining

$$
T^{2} u(z)=-\sum_{j<k}\left(\int_{\partial \mathcal{D}}(G u)(\zeta) d g(\zeta, z) \wedge d \bar{\zeta} \wedge d \zeta[j, k]\right) d \bar{z}[j, k] .
$$

If $M T u=0$ in $\mathcal{D}$ then the restriction of $G u$ to $\partial \mathcal{D}$ extends, by Lemma 8.3, to an antiholomorphic function in $\mathcal{D}$. Hence $T^{2} u(z)=0$ for all $z \in \mathbb{C}^{n} \backslash \overline{\mathcal{D}}$, because $G u$ is orthogonal to the $\partial$-closed differential forms $d g(\zeta, z) \wedge d \bar{\zeta} \wedge d \zeta[j, k]$ by Stokes' formula.

Let $z \in \mathcal{D}$. Then

$$
\begin{aligned}
& \int_{\mathcal{D}} d g \wedge d(G u) \wedge d \bar{\zeta} \wedge d \zeta[j, k] \\
& =\lim _{\varepsilon \rightarrow 0} \int_{\mathcal{D} \backslash\{\zeta:|\zeta-z|<\varepsilon\}} d g \wedge d(G u) \wedge d \bar{\zeta} \wedge d \zeta[j, k] \\
& =-\int_{\partial \mathcal{D}}(G u) d g \wedge d \bar{\zeta} \wedge d \zeta[j, k]+\lim _{\varepsilon \rightarrow 0} \int_{\{\zeta:|\zeta-z|=\varepsilon\}}(G u) d g \wedge d \bar{\zeta} \wedge d \zeta[j, k] \\
& =-\int_{\partial \mathcal{D}}(G u) d g \wedge d \bar{\zeta} \wedge d \zeta[j, k]
\end{aligned}
$$

the latter equality being due to the fact that the restriction of the form $d_{\zeta} g(\zeta, z)$ to $\{\zeta:|\zeta-z|=\varepsilon\}$ vanishes, for $g(\zeta, z)$ is constant on this sphere. Once again we invoke the condition $M T u=0$ in $\mathcal{D}$ and Lemma 8.3 to conclude that $T^{2} u(z)=0$ for all $z \in \mathcal{D}$. Since $T^{2} u$ is of class $H^{1}\left(\mathbb{C}^{n}, \Lambda^{0, n-2}\right)$ we deduce that $T^{2} u$ vanishes in all of $\mathbb{C}^{n}$.

Conversely, let us show that if $T^{2} u=0$ in $\mathbb{C}^{n}$ then $\left.G u\right|_{\partial \mathcal{D}}$ extends to an antiholomorphic function in $\mathcal{D}$. By the above $G u$ is orthogonal under integration over $\partial \mathcal{D}$ to the differential forms

$$
d g(\zeta, z) \wedge d \bar{\zeta} \wedge d \zeta[j, k]
$$

for $z \notin \partial \mathcal{D}$. Since $g(\zeta, z)$ is a fundamental solution of the Laplace equation in $\mathbb{C}^{n}$ (up to a constant factor), differential forms of the type (8.1) are dense in the space of all $\partial$-exact forms of bidegree $(n-2,2)$. By the Hartogs theorem for antiholomorphic functions the restriction $\left.G u\right|_{\partial \mathcal{D}}$ extends to an antiholomorphic function in $\mathcal{D}$. We have thus proved the equivalence of assertions 2) and 3), as desired.

$\square$ The equivalence of assertions 1), 2), 3) and 4) follows from Lemmas 8.3 and 8.4 and from the definition of $U^{s-n}\left(\mathcal{D}, \Lambda^{0, n}\right)$. 
Lemma 8.5 The assertions 4) and 5) of Theorem 8.2 are equivalent.

Proof. Suppose $\left.G u\right|_{\partial \mathcal{D}}$ extends to an antiholomorphic function in $\mathcal{D}$. The potential $G u$ possesses the following properties:

a) $G u \in H^{s-n+2}(\mathcal{D}) \cap H^{2}\left(\mathbb{C}^{n}\right)$;

b) $G u$ is a harmonic function in $\mathbb{C}^{n} \backslash \overline{\mathcal{D}}$;

c) $\Delta G u=c$ in $\mathcal{D}, \Delta$ being the $1 / 4$ multiple of the usual Laplace operator in $\mathbb{C}^{n}$.

It follows that we can take

$$
v=G u \text {. }
$$

Conversely, suppose $u$ fulfills 5 ). Write $v$ by the Green formula in a complex form (cf. Theorem 1.1 in [Kyt95])

$v(z)=\int_{\partial \mathcal{D}}\left(v(\zeta) \overline{U_{0,1}(\zeta, z)}-g(\zeta, z) \sum_{j=1}^{n}(-1)^{j-1} \frac{\partial v}{\partial \zeta_{j}} d \bar{\zeta}[j] \wedge d \zeta\right)+\int_{\mathcal{D}} g(\zeta, z) \Delta v d \bar{\zeta} \wedge d \zeta$

for $z \in \mathcal{D}$. Since $v \in H^{2}\left(\mathbb{C}^{n}\right)$ is harmonic in $\mathbb{C}^{n} \backslash \overline{\mathcal{D}}$, we get from the proof of Lemma 8.3

$$
\int_{\partial \mathcal{D}} v(\zeta) \overline{U_{0,1}(\zeta, z)}=\int_{\partial \mathcal{D}} g(\zeta, z) \sum_{j=1}^{n}(-1)^{j-1} \frac{\partial v}{\partial \zeta_{j}} d \bar{\zeta}[j] \wedge d \zeta
$$

for any $z \in \mathcal{D}$. Hence

$$
\begin{aligned}
v(z) & =\int_{\mathcal{D}} g(\zeta, z) \Delta v(\zeta) d \bar{\zeta} \wedge d \zeta \\
& =(G u)(z),
\end{aligned}
$$

and so $\left.G u\right|_{\partial \mathcal{D}}$ extends to an antiholomorphic function in $\mathcal{D}$.

This proves the theorem.

\section{Regular domains}

In contrast to the extreme cases $q=0$ and $q=n$, where the domain $\mathcal{D}$ is arbitrary, we describe the spaces $U^{s-q}\left(\mathcal{D}, \Lambda^{0, q}\right), 1 \leq q \leq n-1$, for a more restricted class of $\mathcal{D}$. We call them "regular" domains.

By a regular domain we mean any domain $\mathcal{D}$ in $\mathbb{C}^{n}$, such that the cohomology $H^{q}\left(\mathbb{C}^{n} \backslash \overline{\mathcal{D}}, \mathcal{O}\right)$ vanishes for every $q=1, \ldots, n-2$, and for any $\bar{\partial}$-closed form 
$f \in H^{1}\left(\mathbb{C}^{n} \backslash \overline{\mathcal{D}}, \Lambda^{0, q}\right)$ the equation $\bar{\partial} u=f$ has a solution $u \in H^{1}\left(\mathbb{C}^{n} \backslash \overline{\mathcal{D}}, \Lambda^{0, q-1}\right)$ satisfying

$$
\|u\|_{H^{1}\left(\mathbb{C}^{n} \backslash \overline{\mathcal{D}}, \Lambda^{0, q-1}\right)} \leq C\|f\|_{H^{1}\left(\mathbb{C}^{n} \backslash \overline{\mathcal{D}}, \Lambda^{0, q}\right)}
$$

with $C$ a constant independent of $f$. It is well known that strictly pseudoconvex domains in $\mathbb{C}^{n}$ meet these conditions, cf. Theorem 8.7 in [Khe85].

Theorem 9.1 Suppose $\mathcal{D}$ is a regular domain. For any $u \in H^{s-q}\left(\mathcal{D}, \Lambda^{0, q}\right)$ with $0 \leq q \leq n-2$, the condition $M u=0$ in $\mathcal{D}$ is equivalent to $\tau(u)=0$ on $\partial \mathcal{D}$.

Proof. We first show a condition for the $\bar{\partial}$-closedness of the form $M u$.

Lemma 9.2 The form $(M u)^{+}$is $\bar{\partial}$-closed in $\mathcal{D}$ if and only if the form $(M u)^{-}$is $\bar{\partial}$-closed in $\mathbb{C}^{n} \backslash \overline{\mathcal{D}}$.

Proof. Let $\bar{\partial}(M u)^{-}=0$. Then $\nu\left(\bar{\partial}(M u)^{+}\right)=0$ by Theorem 8.1, for

$$
\begin{aligned}
\nu\left(\bar{\partial}(M u)^{+}\right) & =\nu\left((M \bar{\partial} u)^{+}\right) \\
& =\nu\left((M \bar{\partial} u)^{-}\right) \\
& =\nu\left(\bar{\partial}(M u)^{-}\right) \\
& =0
\end{aligned}
$$

on $\partial \mathcal{D}$. Since $(M u)^{+}$is harmonic and, by Lemma $2.1, \bar{\partial}^{*}$-closed in $\mathcal{D}$, we get

$$
\begin{aligned}
\left\|\bar{\partial}(M u)^{+}\right\|^{2} & =\left(\bar{\partial}(M u)^{+}, \bar{\partial}(M u)^{+}\right) \\
& =\left((M u)^{+}, \bar{\partial}^{*} \bar{\partial}(M u)^{+}\right) \\
& =\left((M u)^{+},\left(\Delta-\bar{\partial} \bar{\partial}^{*}\right)(M u)^{+}\right) \\
& =0
\end{aligned}
$$

whence $\bar{\partial}(M u)^{+}=0$ in $\mathcal{D}$. The proof of the converse assertion is quite analogous.

Lemma 9.3 In order that $(M u)^{+}=0$ in $\mathcal{D}$ it is necessary and sufficient that $\tau(u)=-\tau(M u)^{-}$and $\bar{\partial}(M u)^{-}=0$ in $\mathbb{C}^{n} \backslash \overline{\mathcal{D}}$.

Proof. Suppose that $(M u)^{+}=0$ in $\mathcal{D}$. By Theorem 8.1 we see that $\tau(u)=-\tau(M u)^{-}$because $\tau(M u)^{+}=0$. Since $\bar{\partial}(M u)^{+}=0$, Lemma 9.2 yields $\bar{\partial}(M u)^{-}=0$, as desired.

Conversely, let $\tau(u)=-\tau(M u)^{-}$and $\bar{\partial}(M u)^{-}=0$. By Lemma 9.2 we then get $\bar{\partial}(M u)^{+}=0$, and Theorem 8.1 gives $\tau(M u)^{+}=0$. Moreover, Lemma 2.1 shows that $(M u)^{+}=\bar{\partial}^{*} f$ where

$$
f(z)=\int_{\partial \mathcal{D}} u(\zeta) \wedge V_{0, q+1}(\zeta, z)
$$


for $z \in \mathcal{D}$. Applying Lemma 1.1 we thus obtain

$$
\begin{aligned}
\left\|(M u)^{+}\right\|^{2} & =\left((M u)^{+},(M u)^{+}\right) \\
& =\left((M u)^{+}, \bar{\partial}^{*} f\right) \\
& =\left(\bar{\partial}(M u)^{+}, f\right) \\
& =0
\end{aligned}
$$

whence $(M u)^{+}=0$, as desired.

We emphasize that Lemmas 9.2 and 9.3 hold for arbitrary domains $\mathcal{D}$ and differential forms $u$ of any bidegree.

Lemma 9.4 Suppose $f \in H^{1}\left(\mathbb{C}^{n} \backslash \overline{\mathcal{D}}, \Lambda^{0, q}\right)$ satisfies $\bar{\partial} f=0, \bar{\partial}^{*} f=0$ and $\nu(f)=0$. Then $f \equiv 0$.

Proof. Since the domain $\mathcal{D}$ is regular, there is a form $u \in H^{1}\left(\mathbb{C}^{n} \backslash \overline{\mathcal{D}}, \Lambda^{0, q-1}\right)$ such that $\bar{\partial} u=f$. Considering the Hodge scalar product in $\mathbb{C}^{n} \backslash \overline{\mathcal{D}}$ we obtain

$$
\begin{aligned}
\|f\|^{2} & =(f, f) \\
& =(\bar{\partial} u, f) \\
& =\left(u, \bar{\partial}^{*} f\right) \\
& =0
\end{aligned}
$$

whence $f=0$.

End of the proof of Theorem 9.1. Suppose $(M u)^{+}=0$. From Lemma 9.3 it follows that $\tau(u)=-\tau(M u)^{-}$and $\bar{\partial}(M u)^{-}=0$ in $\mathbb{C}^{n} \backslash \overline{\mathcal{D}}$. Furthermore, Theorem 8.1 implies $\nu(M u)^{-}=\nu(M u)^{+}=0$. By Lemma 2.1 we deduce $\bar{\partial}^{*}(M u)^{-}=0$. Applying Lemma 9.4 to the form $f=(M u)^{-}$we derive $(M u)^{-}=0$ in $\mathbb{C}^{n} \backslash \overline{\mathcal{D}}$. Hence $\tau(u)=-\tau(M u)^{-}=0$, as desired. Conversely, if $\tau(u)=0$ then $M u \equiv 0$ by the very definition of the boundary integral $M u$. The proof is complete.

Theorem 9.5 Let $\mathcal{D}$ be a regular domain in $\mathbb{C}^{n}$ and $1 \leq q \leq n-1$. If $u \in H^{s-q}\left(\mathcal{D}, \Lambda^{0, q}\right)$ satisfies $M u=0$ and $M T u=0$ in $\mathcal{D}$ then $u \in U^{s-q}\left(\mathcal{D}, \Lambda^{0, q}\right)$.

The proof of this theorem will follow from two lemmas.

Lemma 9.6 Suppose that $1 \leq q \leq n-1$. If $u \in H^{s-q}\left(\mathcal{D}, \Lambda^{0, q}\right)$ satisfies $M T u=0$ in $\mathcal{D}$ then $T u=0$ in $\mathbb{C}^{n} \backslash \overline{\mathcal{D}}$. 
Proof. Suppose $M T u=0$ in $\mathcal{D}$. Since the degree of the form $M T u$ in $\bar{z}$ is equal to $q-1<n-1$, we obtain $\tau(T u)=0$ on $\partial \mathcal{D}$ by Theorem 9.1. We now invoke Lemma 2.1 to see that

$$
\begin{aligned}
T u(z) & =-\int_{\mathcal{D}} u(\zeta) \wedge U_{0, q}(\zeta, z) \\
& =-\int_{\mathcal{D}} u(\zeta) \wedge \bar{\partial}_{z}^{*} V_{0, q}(\zeta, z) \\
& =-\bar{\partial}^{*} \int_{\mathcal{D}} u(\zeta) \wedge V_{0, q}(\zeta, z) \\
& =-\bar{\partial}^{*} V u
\end{aligned}
$$

for any $z \in \mathbb{C}^{n}$.

The differential form $V u$ has harmonic coefficients in $\mathbb{C}^{n} \backslash \overline{\mathcal{D}}$ since the kernel $V_{0, q}(\zeta, z)$ is harmonic in the external variable $z$. Let us show that $\bar{\partial} T u=0$ in $\mathbb{C}^{n} \backslash \overline{\mathcal{D}}$.

The form $\bar{\partial} T u$ has the following properties:

a) $\tau(\bar{\partial} T u)=0$, for $\tau(\bar{\partial} T u)=\bar{\partial}_{b} \tau(T u)=0$;

b) $\bar{\partial}(\bar{\partial} T u)=0$;

c) $\bar{\partial} T u=-\bar{\partial} \bar{\partial}^{*} V u=\bar{\partial}^{*} \bar{\partial} V u$ in $\mathbb{C}^{n} \backslash \overline{\mathcal{D}}$.

Considering the Hodge inner product over $\mathbb{C}^{n} \backslash \overline{\mathcal{D}}$, we now obtain

$$
\begin{aligned}
\|\bar{\partial} T u\|^{2} & =(\bar{\partial} T u, \bar{\partial} T u) \\
& =\left(\bar{\partial} T u, \bar{\partial}^{*} \bar{\partial} V u\right) \\
& =(\bar{\partial} \bar{\partial} T u, \bar{\partial} V u) \\
& =0
\end{aligned}
$$

whence $\bar{\partial} T u=0$ in $\mathbb{C}^{n} \backslash \overline{\mathcal{D}}$.

On the other hand, the form $T u$ has the following properties:

a) $\tau(T u)=0$;

b) $\bar{\partial} T u=0$;

c) $T u=-\bar{\partial}^{*} V u$ in $\mathbb{C}^{n} \backslash \overline{\mathcal{D}}$.

It follows that

$$
\begin{aligned}
\|T u\|^{2} & =(T u, T u) \\
& =\left(T u,-\bar{\partial}^{*} V u\right) \\
& =-(\bar{\partial} T u, V u) \\
& =0
\end{aligned}
$$

whence $T u=0$ in $\mathbb{C}^{n} \backslash \overline{\mathcal{D}}$, as desired. 
Lemma 9.7 Let $1 \leq q \leq n-1$. If $u \in H^{s-q}\left(\mathcal{D}, \Lambda^{0, q}\right)$ satisfies $M T u=0$ in $\mathcal{D}$ then $T^{2} u=0$ in $\mathbb{C}^{n}$.

Proof. As mentioned in the proof of Lemma 9.6, we have

$$
\begin{aligned}
T u & =-\bar{\partial}^{*} V u \\
& =\star \partial \star V u
\end{aligned}
$$

for all $z \in \mathbb{C}^{n}$. Hence the differential form $T u \wedge F_{1, q-1}(\cdot, z)$ is $\partial$-closed, for

$$
\begin{aligned}
T u \wedge F_{1, q-1}(\cdot, z) & =\star(\partial \star V u) \wedge F_{1, q-1}(\cdot, z) \\
& =(\partial \star V u) \wedge \star F_{1, q-1}(\cdot, z)
\end{aligned}
$$

where $\star F_{1, q-1}(\cdot, z)$ is a differential form with constant coefficients. The equality (2.1) thus gives

$$
\begin{aligned}
T^{2} u(z) & =-\int_{\mathcal{D}} T u(\zeta) \wedge U_{0, q-1}(\zeta, z) \\
& =-\int_{\mathcal{D}} T u(\zeta) \wedge \partial_{\zeta} g(\zeta, z) \wedge F_{1, q-1}(\zeta, z) \\
& =(-1)^{q} \int_{\mathcal{D}} \partial\left(T u(\zeta) \wedge g(\zeta, z) F_{1, q-1}(\zeta, z)\right) \\
& =(-1)^{q} \int_{\mathcal{D}} d\left(T u(\zeta) \wedge g(\zeta, z) F_{1, q-1}(\zeta, z)\right) \\
& =(-1)^{q} \int_{\partial \mathcal{D}} T u(\zeta) \wedge g(\zeta, z) F_{1, q-1}(\zeta, z) \\
& =0
\end{aligned}
$$

for any $z \in \mathbb{C}^{n}$, because $\tau(T u)=0$ on $\partial \mathcal{D}$. In the fourth equality we replaced $\partial$ by the total exterior derivative $d$ since the differential form in parentheses has already $n$ differentials in $\bar{\zeta}$.

Theorem 9.5 has thus been proved.

\section{Characterisation of $U^{s-q}\left(\mathcal{D}, \Lambda^{0, q}\right)$}

We proceed to describe the spaces $U^{s-q}\left(\mathcal{D}, \Lambda^{0, q}\right)$ for $1 \leq q<n-1$. The case $q=n-1$ still falls out the common schema.

Theorem 10.1 Suppose $\mathcal{D}$ is a regular domain in $\mathbb{C}^{n}$ and $1 \leq q<n-1$. In order that $u \in U^{s-q}\left(\mathcal{D}, \Lambda^{0, q}\right)$ it is necessary and sufficient that $\tau(u)=0$ and $u=\Delta v$ in $\mathcal{D}$, where $v \in H^{s-q+2}\left(\mathcal{D}, \Lambda^{0, q}\right) \cap H^{2}\left(\mathbb{C}^{n}, \Lambda^{0, q}\right)$ satisfies $\bar{\partial}^{*} v=0$ and $\bar{\partial}^{*} \bar{\partial} v=0$ in $\mathbb{C}^{n} \backslash \overline{\mathcal{D}}$. 
In particular, $v$ is obviously a harmonic form away from the closure of $\mathcal{D}$ in $\mathbb{C}^{n}$.

Proof. Write

$$
u(z)=\sum_{J} c_{J}(z) d \bar{z}_{J}
$$

where the sum is over all increasing multi-indices $J=\left(j_{1}, \ldots, j_{q}\right)$ of integers $1, \ldots, n$. Let us transform the integral $T u(z)$ for $z \in \mathbb{C}^{n} \backslash \overline{\mathcal{D}}$. By Lemma 2.1 we get

$$
\begin{aligned}
T u(z) & =-\int_{\mathcal{D}} u(\zeta) \wedge U_{0, q}(\zeta, z) \\
& =-\bar{\partial}^{*} \int_{\mathcal{D}} u(\zeta) \wedge V_{0, q}(\zeta, z) .
\end{aligned}
$$

Set

$$
\begin{aligned}
V u(z) & :=\int_{\mathcal{D}} u(\zeta) \wedge V_{0, q}(\zeta, z) \\
& =\sum_{J}\left(\frac{1}{2} \int_{\mathcal{D}} c_{J}(\zeta) g(\zeta, z) d \bar{\zeta} \wedge d \zeta\right) d \bar{z}_{J}
\end{aligned}
$$

for $z \in \mathbb{C}^{n}$. Then $T u=-\bar{\partial}^{*} V u$. The form $V u$ possesses the following properties:

a) $\quad V u \in H^{s-q+2}\left(\mathcal{D}, \Lambda^{0, q}\right) \cap H^{2}\left(\mathbb{C}^{n}, \Lambda^{0, q}\right)$;

b) $V u$ is a harmonic form in $\mathbb{C}^{n} \backslash \overline{\mathcal{D}}$;

c) $\Delta V u=\frac{1}{2} u$ in $\mathcal{D}$.

If $M u=0, M T u=0$ and $q<n-1$, then Theorem 9.1 and Lemma 9.6 imply that $t(u)=0$ and $T u=0$ in $\mathbb{C}^{n} \backslash \overline{\mathcal{D}}$. Hence $\bar{\partial}^{*} V u=0$ in $\mathbb{C}^{n} \backslash \overline{\mathcal{D}}$.

Summarising we conclude that the differential form $v=2 V u$ meets the conclusion of Theorem 10.1, for

$$
\begin{aligned}
0 & =\Delta v \\
& =\bar{\partial} \bar{\partial}^{*} v+\bar{\partial}^{*} \bar{\partial} v \\
& =\bar{\partial}^{*} \bar{\partial} v
\end{aligned}
$$

in $\mathbb{C}^{n} \backslash \overline{\mathcal{D}}$.

Conversely, if $\tau(u)=0$ and $u=\Delta v$ where $v$ satisfies the conditions of the theorem, then $M u=0$ and $v=2 V u$. We show the latter equality by the Green formula in a complex form, applied to the coefficients of $v$.

Namely, write

$$
v(\zeta)=\sum_{J} v_{J}(\zeta) d \bar{\zeta}_{J}
$$


where the sum is over all increasing multi-indices $J$ of length $q$. By Theorem 1.1 of $[$ Kyt95]

$$
v_{J}(z)=\int_{\partial \mathcal{D}}\left(v_{J} U_{0,1}(\cdot, z)-g(\cdot, z) \mu\left(v_{J}\right)\right)+\int_{\mathcal{D}} g(\cdot, z) \Delta v_{J} d \bar{\zeta} \wedge d \zeta
$$

for $z \in \mathcal{D}$, where

$$
\mu(v)=\sum_{j=1}^{n}(-1)^{n+j-1} \frac{\partial v}{\partial \bar{\zeta}_{j}} d \bar{\zeta} \wedge d \zeta[j] .
$$

Since $v_{J} \in H^{2}\left(\mathbb{C}^{n}\right)$ are harmonic functions in $\mathbb{C}^{n} \backslash \overline{\mathcal{D}}$ we get by Stokes' formula

$$
\int_{\partial \mathcal{D}} v_{J} U_{0,1}(\cdot, z)=\int_{\partial \mathcal{D}} g(\cdot, z) \mu\left(v_{J}\right)
$$

for $z \in \mathcal{D}$. Hence it follows that

$$
\begin{aligned}
v_{J}(z) & =\int_{\mathcal{D}} g(\cdot, z) \Delta v_{J} d \bar{\zeta} \wedge d \zeta \\
& =\int_{\mathcal{D}} c_{J} g(\cdot, z) d \bar{\zeta} \wedge d \zeta,
\end{aligned}
$$

$c_{J}$ being the coefficient of $u$ by $d \bar{z}_{J}$. The latter integral is the coefficient of $d \bar{z}_{J}$ in the differential form $V u$ multiplied by 2. Hence $v=2 V u$.

From the properties of $v$ we deduce that

$$
\begin{aligned}
\bar{\partial}^{*} V u & =\frac{1}{2} \bar{\partial}^{*} v \\
& =0
\end{aligned}
$$

in $\mathbb{C}^{n} \backslash \overline{\mathcal{D}}$. Therefore, $T u=-\bar{\partial}^{*} V u=0$ in $\mathbb{C}^{n} \backslash \overline{\mathcal{D}}$, showing the theorem.

\section{The case $q=n-1$}

In the section we describe the remaining spaces $U^{s-n+1}\left(\mathcal{D}, \Lambda^{0, n-1}\right)$ for regular domains.

Theorem 11.1 Assume that $\mathcal{D}$ is a regular domain in $\mathbb{C}^{n}$. In order that $u \in U^{s-n+1}\left(\mathcal{D}, \Lambda^{0, n-1}\right)$ it is necessary and sufficient that

1) $u=\Delta v$ in $\mathcal{D}$, where $v \in H^{s-n+3}\left(\mathcal{D}, \Lambda^{0, n-1}\right) \cap H^{2}\left(\mathbb{C}^{n}, \Lambda^{0, n-1}\right)$ satisfies $\bar{\partial} v=0$ and $\bar{\partial}^{*} v=0$ in $\mathbb{C}^{n} \backslash \overline{\mathcal{D}}$;

2) $\bar{\partial} u=\Delta g d \bar{z}$ in $\mathcal{D}$, where $g \in H^{s-n+2}(\mathcal{D}) \cap H^{2}\left(\mathbb{C}^{n}\right)$ is harmonic in $\mathbb{C}^{n} \backslash \overline{\mathcal{D}}$ and $\left.g\right|_{\partial \mathcal{D}}$ extends to an antiholomorphic function in $\mathcal{D}$. 
Proof. Let $u \in H^{s-n+3}\left(\mathcal{D}, \Lambda^{0, n-1}\right)$ satisfy $M T u=0$ in $\mathcal{D}$. Applying Lemma 9.6 gives $T u=0$ in $\mathbb{C}^{n} \backslash \overline{\mathcal{D}}$. Writing $u$ in the form

$$
u(z)=\sum_{j=1}^{n} c_{j}(z) d \bar{z}[j]
$$

we thus get

$$
\begin{aligned}
T u(z) & =-\int_{\mathcal{D}} u(\zeta) \wedge U_{0, n-1}(\zeta, z) \\
& =-\int_{\mathcal{D}} \sum_{j<k}\left((-1)^{j-1} c_{k}(\zeta) \frac{\partial g}{\partial \zeta_{j}}-(-1)^{k-1} c_{j}(\zeta) \frac{\partial g}{\partial \zeta_{k}}\right) d \bar{\zeta} \wedge d \zeta d \bar{z}[j, k] \\
& =\sum_{j<k}\left((-1)^{j-1} \frac{\partial}{\partial z_{j}}\left(G c_{k}\right)(z)-(-1)^{k-1} \frac{\partial}{\partial z_{k}}\left(G c_{j}\right)(z)\right) d \bar{z}[j, k] \\
& =0
\end{aligned}
$$

for all $z \in \mathbb{C}^{n} \backslash \overline{\mathcal{D}}$, where

$$
\left(G c_{j}\right)(z)=\int_{\mathcal{D}} c_{j}(\zeta) g(\zeta, z) d \bar{\zeta} \wedge d \zeta
$$

For the differential form $v(z)=\sum_{j=1}^{n}\left(G c_{j}\right)(z) d \bar{z}[j]$ the equality (11.1) reduces to $\bar{\partial}^{*} v=0$ in $\mathbb{C}^{n} \backslash \overline{\mathcal{D}}$. Indeed,

$$
\begin{aligned}
\bar{\partial}^{*} v(z) \\
=\frac{1}{2}(-\imath)^{n} \star \partial \sum_{j=1}^{n}(-1)^{j-1}\left(G c_{j}\right) d z_{j} \wedge d \bar{z} \\
=\frac{1}{2}(-\imath)^{n} \star \sum_{j<k}\left((-1)^{k-1} \frac{\partial}{\partial z_{j}}\left(G c_{k}\right)-(-1)^{j-1} \frac{\partial}{\partial z_{k}}\left(G c_{j}\right)\right)\left(d z_{j} \wedge d z_{k}\right) \wedge d \bar{z} \\
=2 \sum_{j<k}\left((-1)^{k-1} \frac{\partial}{\partial z_{k}}\left(G c_{j}\right)-(-1)^{j-1} \frac{\partial}{\partial z_{j}}\left(G c_{k}\right)\right) d \bar{z}[j, k] \\
=0
\end{aligned}
$$

by $(11.1)$.

Our next goal is to establish the equality $\bar{\partial} v=0$ in $\mathbb{C}^{n} \backslash \overline{\mathcal{D}}$. Indeed, the equality $\bar{\partial}^{*} v=0$ means that $\partial \star v=0$. Thus, the differential form $\star v$ is of bidegree $(1, n)$ and $\partial$-closed in the complement of $\overline{\mathcal{D}}$. Since the domain $\mathcal{D}$ is regular this differential form is $\partial$-exact for $n>2$. Hence there exists a form 
$w \in H^{1}\left(\mathbb{C}^{n} \backslash \overline{\mathcal{D}}, \Lambda^{0, n}\right)$ such that $\partial w=\star v$. This form $w$ is actually harmonic in $\mathbb{C}^{n} \backslash \overline{\mathcal{D}}$. Indeed, we have

$$
\begin{aligned}
\partial(\Delta w) & =\Delta(\partial w) \\
& =\Delta(\star v) \\
& =0
\end{aligned}
$$

in $\mathbb{C}^{n} \backslash \overline{\mathcal{D}}$, for the coefficients of $v$ are harmonic functions away from $\overline{\mathcal{D}}$. It follows that $\Delta w$ is an antiholomorphic form in the complement of $\overline{\mathcal{D}}$. By the Hartogs theorem it extends to an integrable antiholomorphic form in all of $\mathbb{C}^{n}$. We now invoke the Liouville theorem for integrable antiholomorphic forms which is proved in much the same way as the Liouville theorem for bounded holomorphic functions. This gives $\Delta w=0$ or $\Delta(\star w)=0$. Since $\star \partial w=(-1)^{n-1} v$ we get

$$
\begin{aligned}
\bar{\partial} v & =(-1)^{n-1} \bar{\partial} \star \partial w \\
& =\bar{\partial} \bar{\partial}^{*}(\star w) \\
& =\Delta(\star w) \\
& =0
\end{aligned}
$$

in $\mathbb{C}^{n} \backslash \overline{\mathcal{D}}$, as desired.

If $n=2$ then (11.1) implies

$$
\frac{\partial}{\partial z_{2}}\left(G c_{1}\right)+\frac{\partial}{\partial z_{1}}\left(G c_{2}\right)=0
$$

and both $G c_{1}$ and $G c_{2}$ are harmonic functions in $\mathbb{C}^{n} \backslash \overline{\mathcal{D}}$. It follows that

$$
\begin{aligned}
\frac{\partial^{2}}{\partial \bar{z}_{1} \partial z_{2}}\left(G c_{1}\right) & =-\frac{\partial^{2}}{\partial \bar{z}_{1} \partial z_{1}}\left(G c_{2}\right) \\
& =\frac{\partial^{2}}{\partial \bar{z}_{2} \partial z_{2}}\left(G c_{2}\right)
\end{aligned}
$$

i.e.,

$$
\frac{\partial}{\partial z_{2}}\left(\frac{\partial}{\partial \bar{z}_{1}}\left(G c_{1}\right)-\frac{\partial}{\partial \bar{z}_{2}}\left(G c_{2}\right)\right)=0
$$

in $\mathbb{C}^{n} \backslash \overline{\mathcal{D}}$. Pick any complex line $z_{1}=z_{1}^{0}$ which does not meet $\overline{\mathcal{D}}$. On this line the function

$$
\frac{\partial}{\partial \bar{z}_{1}}\left(G c_{1}\right)-\frac{\partial}{\partial \bar{z}_{2}}\left(G c_{2}\right)
$$

is antiholomorphic and it tends to zero at the point at infinity. By the Liouville theorem it vanishes on this line. Since the function is real analytic it is equal 
to zero everywhere in the complement of $\overline{\mathcal{D}}$. This just amounts to saying that $v$ is $\bar{\partial}$-closed, i.e., $\bar{\partial} v=0$.

By the properties of the volume potential we see that $v \in H^{2}\left(\mathbb{C}^{n}, \Lambda^{0, n-1}\right)$ and $\Delta v=\chi_{\mathcal{D}} u$ in $\mathbb{C}^{n}$, where $\chi_{\mathcal{D}}$ is the characteristic function of $\mathcal{D}$.

We have thus proved that if $u \in H^{s-n+1}\left(\mathcal{D}, \Lambda^{0, n-1}\right)$ and $M T u=0$ in $\mathcal{D}$, then $u=\Delta v$ in $\mathcal{D}$, where $v \in H^{s-n+3}\left(\mathcal{D}, \Lambda^{0, n-1}\right) \cap H^{2}\left(\mathbb{C}^{n}, \Lambda^{0, n-1}\right)$ satisfies $\bar{\partial} v=0$ and $\bar{\partial}^{*} v=0$ in $\mathbb{C}^{n} \backslash \overline{\mathcal{D}}$.

Conversely, let $u=\Delta v$ with $v$ satisfying the conditions of Theorem 11.1. Write

$$
v(z)=\sum_{j=1}^{n} v_{j}(z) d \bar{z}[j]
$$

where $v_{j} \in H^{s-n+3}(\mathcal{D}) \cap H^{2}\left(\mathbb{C}^{n}\right)$. By the Green formula in a complex form (cf. (10.1)) we get

$$
v_{j}(z)=\int_{\partial \mathcal{D}}\left(v_{j} U_{0,1}(\cdot, z)-g(\cdot, z) \mu\left(v_{j}\right)\right)+\int_{\mathcal{D}} g(\cdot, z) \Delta v_{j} d \bar{\zeta} \wedge d \zeta
$$

for all $z \in \mathcal{D}$ and $j=1, \ldots, n$. Since $v_{j} \in H^{2}\left(\mathbb{C}^{n}\right)$ are harmonic functions in $\mathbb{C}^{n} \backslash \overline{\mathcal{D}}$ we obtain by Stokes' formula

$$
\int_{\partial \mathcal{D}} v_{j} U_{0,1}(\cdot, z)=\int_{\partial \mathcal{D}} g(\cdot, z) \mu\left(v_{J}\right)
$$

for $z \in \mathcal{D}$. Hence it follows that

$$
v_{j}=G c_{j}
$$

in $\mathcal{D}$ for any $j=1, \ldots, n$.

Recall that $c_{j}$ is the coefficient of $d \bar{z}[j]$ in the form $u$. By the above, the equality $\bar{\partial}^{*} v=0$ in the complement of $\overline{\mathcal{D}}$ is equivalent to $T u=0$ there. Since $T u \in H^{2}\left(\mathbb{C}^{n}, \Lambda^{0, n-2}\right)$ the equality $T u=0$ in $\mathbb{C}^{n} \backslash \overline{\mathcal{D}}$ implies $\tau(T u)=0$ whence $M T u=0$ in $\mathcal{D}$.

We have thus proved that the condition $M T u=0$ in $\mathcal{D}$ is equivalent to the assumption 1) of Theorem 11.1.

We now turn to the condition $M u=0$ in $\mathcal{D}$. Namely, we have

$$
\begin{aligned}
M u(z) & =\int_{\partial \mathcal{D}} u(\zeta) \wedge U_{0, n}(\zeta, z) \\
& =\int_{\partial \mathcal{D}}\left(\sum_{k=1}^{n} c_{k}(\zeta) d \bar{\zeta}[k]\right) \wedge\left(\sum_{j=1}^{n}(-1)^{j-1} \frac{\partial g}{\partial \zeta_{j}} d \zeta d \bar{z}[j]\right) \\
& =\sum_{j=1}^{n}(-1)^{j} \frac{\partial}{\partial z_{j}} \int_{\partial \mathcal{D}}\left(\sum_{k=1}^{n} c_{k}(\zeta) g(\zeta, z) d \bar{\zeta}[k] \wedge d \zeta\right) d \bar{z}[j] \\
& =0
\end{aligned}
$$


for $z \in \mathcal{D}$. Hence the condition $M u=0$ in $\mathcal{D}$ is equivalent to the fact that the function

$$
\int_{\partial \mathcal{D}} \sum_{k=1}^{n} c_{k}(\zeta) g(\zeta, z) d \bar{\zeta}[k] \wedge d \zeta
$$

is antiholomorphic in the domain $\mathcal{D}$. Applying Stokes' formula to this integral we get

$$
\begin{aligned}
\int_{\partial \mathcal{D}} \sum_{k=1}^{n} c_{k}(\zeta) g(\zeta, z) d \bar{\zeta}[k] \wedge d \zeta & =\int_{\mathcal{D}} \sum_{k=1}^{n}(-1)^{k-1} \frac{\partial}{\partial \bar{\zeta}_{k}}\left(c_{k}(\zeta) g(\zeta, z)\right) d \bar{\zeta} \wedge d \zeta \\
& =\int_{\mathcal{D}} \sum_{k=1}^{n}(-1)^{k-1} \frac{\partial c_{k}}{\partial \bar{\zeta}_{k}} g(\zeta, z) d \bar{\zeta} \wedge d \zeta
\end{aligned}
$$

for any $z \in \mathbb{C}^{n} \backslash \overline{\mathcal{D}}$, since the integral

$$
\int_{\mathcal{D}} \sum_{k=1}^{n}(-1)^{k-1} c_{k}(\zeta) \frac{\partial}{\partial \bar{\zeta}_{k}} g(\zeta, z) d \bar{\zeta} \wedge d \zeta=\sum_{k=1^{n}}(-1)^{k} \frac{\partial}{\partial \bar{z}_{k}}\left(G c_{k}\right)(z)
$$

vanishes by the closedness of the differential form

$$
v(z)=\sum_{j=1}^{n}\left(G c_{j}\right)(z) d \bar{z}[j]
$$

Summarising we deduce that the condition $M u=0$ in $\mathcal{D}$ just amounts to saying that the potential

$$
\begin{aligned}
(G \bar{\partial} u)(z) & =\int_{\mathcal{D}} \sum_{k=1}^{n}(-1)^{k-1} \frac{\partial c_{k}}{\partial \bar{\zeta}_{k}} g(\zeta, z) d \bar{\zeta} \wedge d \zeta \\
& =\int_{\mathcal{D}} \bar{\partial} u(\zeta) \wedge g(\zeta, z) d \zeta
\end{aligned}
$$

when restricted to $\partial \mathcal{D}$, extends to an antiholomorphic function in $\mathcal{D}$. We can thus take $g=G \bar{\partial} u$ in all of $\mathbb{C}^{n}$. Obviously, this function meets all assumptions of 2).

Conversely, if $\bar{\partial} u=\Delta g d \bar{z}$ in $\mathcal{D}$, where $g \in H^{s-n+2}(\mathcal{D}) \cap H^{2}\left(\mathbb{C}^{n}\right)$ is harmonic in $\mathbb{C}^{n} \backslash \overline{\mathcal{D}}$ and $\left.g\right|_{\partial \mathcal{D}}$ extends to an antiholomorphic function in $\mathcal{D}$, then $g=G \bar{\partial} u$ in $\mathcal{D}$ by the Green formula. It follows that the restriction of $G \bar{\partial} u$ to $\partial \mathcal{D}$ extends to an antiholomorphic function in $\mathcal{D}$. Hence $M u=0$ in $\mathcal{D}$, showing the theorem. 


\section{Concluding remarks}

The exact subcomplex of the Dolbeault complex introduced in Section 4 is by no means unique. It is of interest because of its link to the integral formula of Koppelman, cf. Lemma 2.3.

As but one example of other exact subcomplexes of the Dolbeault complex we show here a subcomplex closely related to the $\bar{\partial}$-Neumann problem. While this latter problem is subelliptic only in strictly pseudoconvex domains on complex manifolds, the subcomplex is exact in any domain with smooth boundary.

More precisely, for $s=2,3, \ldots$, we introduce the space $\mathfrak{U}^{s}\left(\mathcal{D}, \Lambda^{0, q}\right)$ to consists of all $u \in H^{s}\left(\mathcal{D}, \Lambda^{0, q}\right)$ such that

$$
\begin{aligned}
\tau(u) & =0, \\
\nu(u) & =0, \\
\nu(\bar{\partial} u) & =0
\end{aligned}
$$

on $\partial \mathcal{D}$, and $\bar{\partial}^{*} u=\Delta v$ in $\mathcal{D}$, with $v \in H^{s+1}\left(\mathcal{D}, \Lambda^{0, q-1}\right) \cap \stackrel{\circ}{H^{2}}\left(\mathcal{D}, \Lambda^{0, q-1}\right)$.

Lemma 12.1 As described above, the differential form $v$ satisfies $\bar{\partial}^{*} v=0$ in $\mathcal{D}$.

Proof. Indeed, from $\bar{\partial}^{*} u=\Delta v$ it follows that $\bar{\partial}^{*} \bar{\partial} \bar{\partial}^{*} v=0$ in $\mathcal{D}$. Combining this with $\tau\left(\bar{\partial}^{*} v\right)=0$ on $\partial \mathcal{D}$ we get $\bar{\partial} \bar{\partial}^{*} v=0$ in $\mathcal{D}$. Using once again the condition $\tau\left(\bar{\partial}^{*} v\right)=0$ on $\partial \mathcal{D}$ we in turn obtain $\bar{\partial}^{*} v=0$ in $\mathcal{D}$, as desired.

Lemma 12.2 Suppose that $u \in \mathfrak{U}^{s}\left(\mathcal{D}, \Lambda^{0, q}\right)$ is $\bar{\partial}$-closed in $\mathcal{D}$. Then we have $u=\bar{\partial} v$, $v$ being as above.

Proof. Indeed, the equality $\bar{\partial}^{*} u=\Delta v$ implies, by Lemma 12.1, that $\bar{\partial}^{*}(u-\bar{\partial} v)=0$ in $\mathcal{D}$. On the other hand, we have $\bar{\partial}(u-\bar{\partial} v)=0$ in $\mathcal{D}$, for $u$ is $\bar{\partial}$-closed in $\mathcal{D}$. Since both $\tau(u-\bar{\partial} v)$ and $\nu(u-\bar{\partial} v)$ vanish on the boundary of $\mathcal{D}$, it follows from the uniqueness theorem that $u-\bar{\partial} v \equiv 0$ in $\mathcal{D}$, which completes the proof.

Invoking these lemmas we easily arrive at the following theorem analogous to Theorem 4.1 .

Theorem 12.3 Suppose $s>n$. The following sequence of Hilbert spaces is an exact complex:

$$
0 \longrightarrow \mathfrak{U}^{s}\left(\mathcal{D}, \Lambda^{0,0}\right) \stackrel{\bar{\partial}}{\longrightarrow} \mathfrak{U}^{s-1}\left(\mathcal{D}, \Lambda^{0,1}\right) \stackrel{\bar{\partial}}{\longrightarrow} \ldots \stackrel{\bar{\partial}}{\longrightarrow} \mathfrak{U}^{s-n}\left(\mathcal{D}, \Lambda^{0, n}\right) \longrightarrow 0 .
$$


Since $u \mapsto\{\tau(u), \nu(\bar{\partial} u)\}$ is a Dirichlet system of order 1 on $\partial \mathcal{D}$, we obviously have

$$
\begin{aligned}
U^{s}\left(\mathcal{D}, \Lambda^{0,0}\right) & =H^{s}(\mathcal{D}) \cap \stackrel{\circ}{H}^{1}(\mathcal{D}), \\
\mathfrak{U}^{s}\left(\mathcal{D}, \Lambda^{0,0}\right) & =H^{s}(\mathcal{D}) \cap \stackrel{\circ}{H}^{2}(\mathcal{D}) .
\end{aligned}
$$




\section{References}

[AD83] L. A. Aizenberg and Sh. A. Dautov, Differential Forms Orthogonal to Holomorphic Functions or Forms, and Their Properties, AMS, Providence, R.I., 1983.

[Agr97] M. S. Agranovich, Elliptic Boundary Problems, In: Partial Differential Equations IX, Encyclopaedia of Mathematical Sciences, Vol. 79, Springer, Berlin et al., 1997, 1-144.

[AB64] M. F. АтाYAн and R. Bотт, The index problem for manifolds with boundary, In: Coll. Differential Analysis, Tata Institute Bombay, Oxford University Press, Oxford, 1964, 175-186.

[AB67] M. F. Атіуан and R. Bотт, A Lefschetz fixed point formula for elliptic complexes. I, Ann. Math. 86 (1967), no. 2, 374-407.

[APS75] M. F. Atiyah, V. K. Patodi, and I. M. Singer, Spectral asymmetry and Riemannian geometry. I, Math. Proc. Camb. Phil. Soc. 77 (1975), 43-69.

[BdM71] L. Boutet DE Monvel, Boundary problems for pseudo-differential operators, Acta Math. 126 (1971), no. 1-2, 11-51.

[Cal63] A. P. CALDERón, Boundary value problems for elliptic equations, In: Outlines of the Joint Soviet-American Symposium on PDE's, Novosibirsk, 1963, 303-304.

[Chi75] E. M. Chirka, Analytic representation of $C R$-functions, Mat. Sb. 98 (1975), no. 4, 591-623.

[Dyn72] A. S. Dynin, Elliptic boundary problems for pseudo-differential complexes, Funkts. Analiz 6 (1972), no. 1, 75-76.

[Gru86] G. GrubB, Functional Calculus of Pseudodifferential Boundary Problems, Birkhäuser Verlag, Basel et al., 1986.

[Hed81] L. I. HedBerg, Spectral synthesis in Sobolev spaces and uniqueness of solutions of the Dirichlet problem, Acta Math. 147 (1981), 235264.

[Khe85] G. M. Khenkin, The method of integral representation in complex analysis, In: Current Problems of Mathematics, Fundamental Directions, Vol. 7, VINITI, Moscow, 1985, 23-124. 
[KR65] J. J. KoHn and H. Rossi, On the extension of holomorphic functions from the boundary of a complex manifold, Ann. Math. 81 (1965), no. 3, 451-472.

[Kon67] V. A. Kondrat'Ev, Boundary value problems for elliptic equations in domains with conical points, Trudy Mosk. Mat. Obshch. 16 (1967), 209-292.

[Kop67] W. Koppelman, The Cauchy integral for differential forms, Bull. Amer. Math. Soc. 73 (1967), no. 4, 554-556.

[Kyt95] A. M. Kytmanov, The Bochner-Martinelli Integral, and Its Applications, Birkhäuser Verlag, Basel et al., 1995.

[PS80] V. Pillat and B.-W. Schulze, Elliptische Randwert-Probleme für Komplexe von Pseudodifferentialoperatoren, Math. Nachr. 94 (1980), 173-210.

[RS82] St. Rempel and B.-W. Schulze, Index Theory of Elliptic Boundary Problems, Akademie-Verlag, Berlin, 1982.

[Roi01] Ya. A. Roitberg, Elliptic Boundary Value Problems in Generalized Functions, Kluwer Academic Publishers, Dordrecht NL, 1996.

[Rom78] A. V. Romanov, Spectral analysis of Martinelli-Bochner integral for a ball in $\mathbb{C}^{n}$, and its application, Funkts. Analiz 12 (1978), no. 3, $86-87$.

[Schu01] B.-W. Schulze, An algebra of boundary value problems not requiring Shapiro-Lopatinskij conditions, J. Funct. Anal. 179 (2001), 374-408.

[See69] R. SEeley, Topics in pseudo-differential operators, In: Cremonese (eds), Pseudo-Differential Operators, CIME, Roma, 1969, 167-306.

[Spa66] E. H. Spanier, Algebraic Topology, McGraw-Hill, New York, 1966.

[Tar90] N. Tarkhanov, Parametrix Method in the Theory of Differential Complexes, Nauka, Novosibirsk, 1990.

[Ven72] V. Venugopalkrishna, Fredholm operators associated with strongly pseudoconvex domains in $\mathbb{C}^{n}$, J. Funct. Anal. 9 (1972), 349373.

[VE65] M. I. VISHIK and G. I. Eskin, Convolution equations in a bounded region, Russian Math. Surveys 20 (1965), no. 3, 85-152. 
[Wel73] R. Wells, Differential Analysis on Complex Manifolds, PrenticeHall, Englewood Cliffs, N.J., 1973.

(A. Kytmanov) Krasnoyarsk State University, Pr. Svobodnyi 79, 660041 Krasnoyarsk, Russia E-mail address: kytmanov@math.kgu.krasnoyarsk.su

(S. Myslivets) Krasnoyarsk State University, pr. Svobodnyi 79, 660041 Krasnoyarsk, Russia E-mail address: simona@math.kgu.krasnoyarsk.su

(B.-W. Schulze) Universität Potsdam, Institut für Mathematik, Postfach 6015 53, 14415 PotsDAM, GERMANY

E-mail address: schulze@math.uni-potsdam.de

(N. Tarkhanov) Universität Potsdam, Institut für Mathematik, Postfach 6015 53, 14415 PotsDAm, Germany

E-mail address: tarkhanov@math.uni-potsdam.de 